\title{
\begin{tabular}{l|l} 
Mitraries & DSpace@MIT
\end{tabular}
}

\author{
MIT Open Access Articles
}

\section{HUBBLE SPACE TELESCOPE MEASURES OF MASS ACCRETION RATES IN THE ORION NEBULA CLUSTER}

The MIT Faculty has made this article openly available. Please share how this access benefits you. Your story matters.

Citation: Manara, C. F., M. Robberto, N. Da Rio, G. Lodato, L. A. Hillenbrand, K. G. Stassun, and D. R. Soderblom. “HUBBLE SPACE TELESCOPE MEASURES OF MASS ACCRETION RATES IN THE ORION NEBULA CLUSTER.” The Astrophysical Journal 755, no. 2 (August 6, 2012): 154. (C) 2012 The American Astronomical Society

As Published: http://dx.doi.org/10.1088/0004-637x/755/2/154

Publisher: IOP Publishing

Persistent URL: http://hdl.handle.net/1721.1/95503

Version: Final published version: final published article, as it appeared in a journal, conference proceedings, or other formally published context

Terms of Use: Article is made available in accordance with the publisher's policy and may be subject to US copyright law. Please refer to the publisher's site for terms of use. 


\title{
HUBBLE SPACE TELESCOPE MEASURES OF MASS ACCRETION RATES IN THE ORION NEBULA CLUSTER
}

\author{
C. F. Manara ${ }^{1,2,3}$, M. Robberto ${ }^{1}$, N. Da Rio ${ }^{4}$, G. Lodato ${ }^{3}$, L. A. Hillenbrand ${ }^{5}$, \\ K. G. Stassun ${ }^{6,7,8}$, AND D. R. Soderblom ${ }^{1}$ \\ ${ }^{1}$ Space Telescope Science Institute, 3700 San Martin Drive, Baltimore, MD 21218, USA; cmanara@eso.org \\ ${ }^{2}$ European Southern Observatory, Karl Schwarzschild-Str. 2, D-85748 Garching, Germany \\ ${ }^{3}$ Dipartimento di Fisica, Universitá Degli Studi di Milano, Via Celoria, 16, Milano 20133, Italy \\ ${ }^{4}$ European Space Agency, Keplerlaan 1, 2200 AG Noordwijk, The Netherlands \\ ${ }^{5}$ Department of Astronomy, California Institute of Technology, 1200 East California Boulervard, Pasadena, CA 91125, USA \\ ${ }^{6}$ Department of Physics \& Astronomy, Vanderbilt University, 6301 Stevenson Center Ln., Nashville, TN 37235, USA \\ ${ }^{7}$ Department of Physics, Fisk University, 1000 17th Avenue North, Nashville, TN 37208, USA \\ ${ }^{8}$ Massachusetts Institute of Technology, Department of Physics, 77 Massachusetts Avenue, Cambridge, MA 02139, USA \\ Received 2012 May 3; accepted 2012 June 19; published 2012 August 6
}

\begin{abstract}
The present observational understanding of the evolution of the mass accretion rates $\left(\dot{M}_{\text {acc }}\right)$ in pre-main-sequence stars is limited by the lack of accurate measurements of $\dot{M}_{\text {acc }}$ over homogeneous and large statistical samples of young stars. Such observational effort is needed to properly constrain the theory of star formation and disk evolution. Based on Hubble Space Telescope/WFPC2 observations, we present a study of $\dot{M}_{\text {acc }}$ for a sample of $\sim 700$ sources in the Orion Nebula Cluster, ranging from the hydrogen-burning limit to $M_{*} \sim 2 M_{\odot}$. We derive $\dot{M}_{\text {acc }}$ from both the $U$-band excess and the $\mathrm{H} \alpha$ luminosity $\left(L_{\mathrm{H} \alpha}\right)$, after determining empirically both the shape of the typical accretion spectrum across the Balmer jump and the relation between the accretion luminosity $\left(L_{\text {acc }}\right)$ and $L_{\mathrm{H} \alpha}$, which is $L_{\mathrm{acc}} / L_{\odot}=(1.31 \pm 0.03) \cdot L_{\mathrm{H} \alpha} / L_{\odot}+(2.63 \pm 0.13)$. Given our large statistical sample, we are able to accurately investigate relations between $\dot{M}_{\text {acc }}$ and the parameters of the central star such as mass and age. We clearly find $\dot{M}_{\text {acc }}$ to increase with stellar mass and decrease over evolutionary time, but we also find strong evidence that the decay of $\dot{M}_{\text {acc }}$ with stellar age occurs over longer timescales for more massive PMS stars. Our best-fit relation between these parameters is given by $\log \left(\dot{M}_{\text {acc }} / M_{\odot} \mathrm{yr}\right)=(-5.12 \pm 0.86)-(0.46 \pm 0.13) \cdot \log (t / \mathrm{yr})-$ $(5.75 \pm 1.47) \cdot \log \left(M_{*} / M_{\odot}\right)+(1.17 \pm 0.23) \cdot \log (t / \mathrm{yr}) \cdot \log \left(M_{*} / M_{\odot}\right)$. These results also suggest that the similarity solution model could be revised for sources with $M_{*} \gtrsim 0.5 M_{\odot}$. Finally, we do not find a clear trend indicating environmental effects on the accretion properties of the sources.
\end{abstract}

Key words: accretion, accretion disks - protoplanetary disks - stars: formation - stars: pre-main sequence

Online-only material: animation, color figures, machine-readable tables

\section{INTRODUCTION}

During the pre-main-sequence (PMS) phase of stellar evolution, the interaction between the forming star and the surrounding disk is regulated by the accretion of disk material along the field lines of the stellar magnetosphere (Hartmann et al. 1998). The gravitational energy released as the material falls along accretion columns and hits the stellar surface creates a characteristic shock spectrum (Calvet \& Gullbring 1998), with excess emission especially strong in the Balmer continuum and recombination lines (Gullbring et al. 1998; Calvet \& Gullbring 1998). The relative accretion luminosity $\left(L_{\text {acc }}\right)$ can be measured with spectroscopic (Valenti et al. 1993; Herczeg \& Hillenbrand 2008) or photometric (Gullbring et al. 1998; Robberto et al. 2004; De Marchi et al. 2010) methods. The mass accretion rate, $\dot{M}_{\text {acc }}$, is then estimated through the relation (Hartmann et al. 1998)

$$
\dot{M}_{\mathrm{acc}}=\frac{L_{\mathrm{acc}} R_{*}}{0.8 G M_{*}},
$$

where $R_{*}$ and $M_{*}$ are the radius and the mass of the star, respectively, and the factor 0.8 accounts for the assumption that the infall originates at a magnetospheric radius $R_{\mathrm{m}}=5$ (Shu et al. 1994).

The mass accretion rate generally decreases over time during the first few Myr of PMS stellar evolution, as the circumstellar disks disperse their gaseous content on a timescale of $\sim 3-5 \mathrm{Myr}$ (Haisch et al. 2001; Dahm 2005; Fedele et al. 2010). Moreover, $\dot{M}_{\text {acc }}$ is expected to scale with stellar mass. The evolution of $\dot{M}_{\text {acc }}$ versus time as a function of the stellar and disk mass represents a key aspect of PMS evolution and planet formation. Hartmann et al. (1998) showed that while typical $\dot{M}_{\text {acc }}$ values, at any given stellar age, can differ star by star of up to about two orders of magnitude, on average they decrease exponentially with the stellar age $t$, i.e., $\dot{M}_{\text {acc }} \propto t^{-\eta}$ with $\eta \sim 1.5$. Several studies, targeting different young stellar clusters, have confirmed this general trend (e.g., Robberto et al. 2004; Sicilia-Aguilar et al. 2010), but the uncertainties in the age estimate (Hartmann et al. 1997; Baraffe \& Chabrier 2010) and the scarcity of rich and homogeneous samples limit the accurate assessment of this dependence.

For what concerns the stellar mass dependence of $\dot{M}_{\text {acc }}$, Muzerolle et al. (2003) found that $\dot{M}_{\text {acc }} \propto M_{*}^{b}$ with $b=2$, although recent studies (e.g., Rigliaco et al. 2011) suggest smaller values of $b$. Due to the large spread in the $\dot{M}_{\text {acc }}$ values for a given $M_{*}$ (up to two orders of magnitudes; Rigliaco et al. 2011), this second relation has also never been accurately constrained. Although observational uncertainties and intrinsic variability will always contribute in scattering the measured mass accretion rates, accurate measurements of $\dot{M}_{\text {acc }}$ on a large sample of PMS stars may allow us to assess more precisely the value of the two power-law exponents $(\eta$ and $b)$. This is 
a critical step, as they can be tied to the theoretical model for the disk evolution and structure, providing unique constraints to the initial conditions of planet formation (e.g., Alexander \& Armitage 2006; Lodato 2008).

As the nearest ( $d \sim 414 \pm 7$ pc; Menten et al. 2007) site of massive star formation, the Orion Nebula Cluster (ONC) provides a standard benchmark for star formation studies. The ONC population has been studied in depth, and determination of the individual stellar parameters are available for a large fraction of members. In particular, Hillenbrand (1997), Da Rio et al. (2010), and Da Rio et al. (2012) have used both spectroscopic and photometric techniques to derive the spectral type of more than 1700 ONC sources. The corresponding initial mass function, ranging from the brown dwarfs (BD) regime to the O6 star $\theta^{1}$ Ori-C, peaks at $0.2-0.3 M_{\odot}$ (Da Rio et al. 2012), while the cluster mean age is $\sim 2.2 \mathrm{Myr}$, with evidence for an age spread of the order of $\sim 2$ Myr (Reggiani et al. 2011). Given the wealth of information on the individual cluster members, the ONC is ideally suited to conduct an extensive study of the mass accretion process in PMS stars.

In this paper, we present the results of such a study based on the Hubble Space Telescope (HST)/WFPC2 survey of the ONC (GO 10246, PI: M. Robberto). Both $U$-band and $\mathrm{H} \alpha$ data are used to estimate $\dot{M}_{\text {acc }}$ for $\sim 700$ sources and to analyze the relations between this parameter and the main stellar parameters (age, $M_{*}$ ). In Section 2, we illustrate the observation, data reduction, and analysis of our WFPC2 photometry. Section 3 presents our derived modeling of the photospheric colors for our sources and for the typical accretion spectrum, and the methods used to obtain the $L_{\text {acc }}$ based on our data, both using our UBI diagram method and from the $\mathrm{H} \alpha$ photometry, while in Section 4 we present the derived quantities $\left(L_{\text {acc }}, \dot{M}_{\text {acc }}\right)$ obtained both from the measurement of the $U$ band or from $L_{\mathrm{H} \alpha}$; in Section 5, we study the evolution of these parameters as a function the main stellar parameter. Finally, in Section 6 we summarize our conclusions.

\section{OBSERVATIONS AND DATA REDUCTION}

\subsection{Observations}

In this work, we consider the HST observations obtained for the HST Treasury Program on the ONC (Robberto et al. 2012). In particular, we use the WFPC2 observations carried out between 2004 October and 2005 April in the filters $F 336 W$, $F 439 W, F 656 N$, and $F 814 W$. One of the advantages of this data set is the nearly simultaneous (within less than one hour) imaging obtained in subsequent exposures in $U(F 336 W)$ and $\mathrm{H} \alpha(F 656 N)$ bands, which, as will be described in the next sections, both allow an estimate of the accretion luminosity.

Robberto et al. (2012) details the data acquisition and reduction strategy of all the photometry obtained within the Treasury Program; here we summarize only the key aspects of the WFPC2 data set, which are most relevant to our study. The $F 336 \mathrm{~W}$ filter is roughly analogous to the standard Strömgren $u$ filter, with a long wavelength cutoff at $\lambda \simeq 3600 \AA$, shortward the Balmer jump. This WFPC2 filter, however, is affected by "red leak," which is a residual transmission at longer wavelengths $(\lambda \simeq$ $7300 \AA$ A). This effect alters the measured $U$-band fluxes for red sources, and in Appendix A we explain how the correction for this leak is performed. We hereafter refer to the corrected $U$ band as $U$, using the notation $U_{\text {noleak }}$ only when needed, for clarity. The $F 439 W$ filter closely matches the standard Johnson $B$ passband, while the $F 656 N$ filter is a narrowband filter centered on the $\mathrm{H} \alpha$ line, narrow enough to exclude the adjacent [N II] doublet lines. The last filter, $F 814 \mathrm{~W}$, is similar to the Cousin Ic filter; it is fundamental for our study both to determine the red leak contamination of the $U$ band and to derive the bolometric luminosity of the ONC members. The exposure times for each field are $800 \mathrm{~s}$ in $F 336 \mathrm{~W}, 80 \mathrm{~s}$ in $F 439 \mathrm{~W}, 400 \mathrm{~s}$ in $F 656 \mathrm{~N}$, and 10 s exposure in $F 814 W$.

Aperture photometry was obtained by choosing aperture radii of $0^{\prime \prime} .1$ and 0.5 , corresponding to 5 and 11 pixels on the WF1 (PC) and 2 and 5 pixels on WF2-4 chips. The sky annulus has always been taken between $1^{\prime \prime}$. and $1^{\prime \prime} .5$, corresponding to 20 and 30 pixels with WF1 and 10 and 15 pixels with WF2-4. At the time the observations were carried out, the WFPC2 instrument had already accumulated about 11 years of total radiation dose in space environment, and therefore was affected by non-negligible charge transfer efficiency (CTE) losses. The brightness of the Orion Nebula background, especially in our four broadband filters and at the center of the region, mitigates the problem but still we had to apply a CTE correction to the measured counts following the recipe of Dolphin (2009). We estimated CTE correction errors using a Monte Carlo propagation of the errors terms in the equation of Dolphin (2009).

The final photometric errors have been derived by adding in quadrature the errors associated with the measured counts, zero point, CTE correction, aperture correction to the infinity and, in the case of the $0^{\prime \prime} 1$ apertures, an extra aperture correction to the 0.5 radius. This last term is usually dominating, ensuring that the 0.5 photometry is generally more accurate and therefore preferred, except for the faintest sources detected in very few pixels.

\subsection{Source Selection}

Our WFPC2 photometric catalog includes 1643 sources in total with detection in at least one of the four bands. Among these, we have removed 27 known proplyds, identified by Ricci et al. (2008) from the ACS imaging, since the fluxes of these are potentially contaminated by circumstellar emission. We have also eliminated 144 close binary systems ( $d \lesssim 3$ pix $\sim 75-100$ AU), resolved either in the ACS or the WFPC2 imaging, where the spectral-type classification is more uncertain. For well-separated visual binaries, we carefully inspected the individual sources to rule out possible matching errors between the photometry in different bands.

In the remaining sample, $U$-band photometry is available for 1021 stars ( $\sim 60 \%$ of the total), among these, 897 sources ( $\sim 55 \%$ ) have been detected in all $U, B$, and $I$ bands. Finally, for 1342 sources $(\sim 80 \%) \mathrm{H} \alpha$ photometry is available.

\section{METHOD}

We follow the approach introduced by Da Rio et al. (2010) to obtain simultaneously an estimate of extinction $\left(A_{V}\right)$ and accretion $\left(L_{\mathrm{acc}} / L_{\mathrm{tot}}\right.$, where $\left.L_{\mathrm{tot}}=L_{*}+L_{\mathrm{acc}}\right)$ for individual sources from the photometry in three optical bands together with the $T_{\text {eff }}$ of the source. The procedure assumes that on a color-color diagram the displacement of the observed sources from the theoretical isochrone is due to a combination of extinction and accretion. First of all, accretion excess causes a shift in the colors toward bluer values, and then extinction moves the resulting color along the reddening direction. In particular, in a two-color diagram, accretion displaces the sources along curves that start from the isochrone and converge to a point defined by the colors of the pure accretion spectrum. If $T_{\text {eff }}$ of 


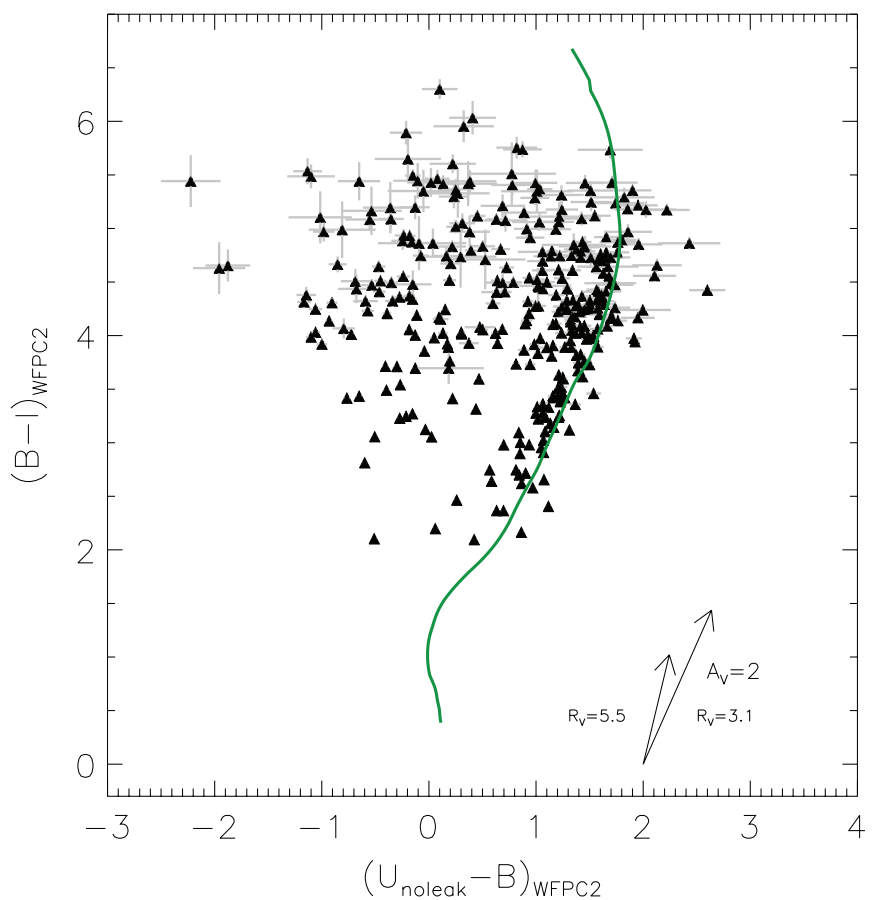

Figure 1. Our $U B I 2 \mathrm{CD}$; the thick line is our calibrated isochrone valid for the ONC. The arrows indicate two reddening vectors, corresponding to $A_{V}=2$, for two different values of $R_{V}$ assuming the extinction law of Cardelli et al. (1989). (A color version of this figure is available in the online journal.)

a star is known, e.g., from spectroscopy, the combination of $A_{V}$ and $L_{\text {acc }} / L_{\text {tot }}$ necessary to reproduce the observed colors is unequivocally found.

In Da Rio et al. (2010), a $B V I$ two-color diagram was used; here, instead we construct the $U_{\text {noleak }}-B$ versus $B-I$ two-color diagram. Since $U$ band is far more sensitive to accretion than $B$, this allows us to measure $L_{\text {acc }} / L_{\text {tot }}$ with a significantly higher accuracy than in Da Rio et al. (2010). We will refer from now on to this two-color diagram as the $2 \mathrm{CD}$.

\subsection{The Two-color Diagram: Extinction and Accretion Luminosity}

We consider the measured $T_{\text {eff }}$ for the ONC members from Da Rio et al. (2012), and limit ourselves to the sources detected in all the three WFPC2 bands and where the error on the $U_{\text {noleak }}$ values is $\lesssim 0.3$ mag. ${ }^{9}$ Our sample is then composed by 339 ONC members. Figure 1 shows the observed 2CD of this sample; the solid line represents our calibrated isochrone (see Section 3.1.1 for details).

To model the extinction, we assume the reddening law of Cardelli et al. (1989) with typical galactic reddening parameter $R_{V}=3.1$. Some authors have suggested a larger value of $R_{V}=5.5$ for the ONC (e.g., Costero \& Peimbert 1970) and we show this as well in Figure 1. Da Rio et al. (2010), however, found $R_{V}=3.1$ to be more appropriate, on average, for the ONC population. Therefore, we will adopt this value for our analysis.

\footnotetext{
9 The photometrical errors in all the bands $(U, B, I)$, calculated as in Section 2.1, are always lower than $0.2 \mathrm{mag}$, but the correction for the red leak (see Appendix A) raises the final error on the measure to values up to $\sim 0.29$ mag in 15 objects.
}

Table 1

Intrinsic Colors for the WFPC2 Bands $F 336 W(U), F 439 W(B)$, and $F 814 W$ $(I)$ and Bolometric Correction for the $F 814 W(I)$ Band Obtained Assuming Allard et al. (2011) Models

\begin{tabular}{|c|c|c|c|c|}
\hline $\begin{array}{l}\text { Spectral } \\
\text { Type }\end{array}$ & $\begin{array}{l}T_{\text {eff }} \\
(\mathrm{K})\end{array}$ & $\begin{array}{c}(U-B) \\
(\mathrm{mag})\end{array}$ & $\begin{array}{c}(B-I) \\
(\mathrm{mag})\end{array}$ & $\begin{array}{l}\left(\mathrm{BC}_{I}\right) \\
(\mathrm{mag})\end{array}$ \\
\hline A5 & 8200 & 0.126 & 0.285 & -0.450 \\
\hline A7 & 7850 & 0.106 & 0.424 & -0.365 \\
\hline F0 & 7200 & 0.066 & 0.673 & -0.236 \\
\hline $\mathrm{F} 2$ & 6890 & 0.017 & 0.809 & -0.180 \\
\hline F5 & 6440 & -0.013 & 1.022 & -0.105 \\
\hline F8 & 6200 & -0.002 & 1.151 & -0.068 \\
\hline G0 & 6030 & 0.022 & 1.247 & -0.044 \\
\hline $\mathrm{G} 2$ & 5860 & 0.054 & 1.352 & -0.021 \\
\hline G5 & 5770 & 0.075 & 1.406 & -0.012 \\
\hline G8 & 5570 & 0.142 & 1.530 & 0.007 \\
\hline $\mathrm{K} 1$ & 5080 & 0.457 & 1.871 & 0.032 \\
\hline $\mathrm{K} 2$ & 4900 & 0.597 & 2.024 & 0.033 \\
\hline $\mathrm{K} 3$ & 4730 & 0.710 & 2.188 & 0.027 \\
\hline K4 & 4590 & 0.790 & 2.342 & 0.017 \\
\hline K5 & 4350 & 0.945 & 2.636 & -0.010 \\
\hline K7 & 4060 & 1.111 & 2.997 & -0.055 \\
\hline M0 & 3850 & 1.234 & 3.272 & -0.108 \\
\hline M1 & 3705 & 1.313 & 3.460 & -0.159 \\
\hline M2 & 3560 & 1.412 & 3.646 & -0.218 \\
\hline M3 & 3415 & 1.543 & 3.890 & -0.288 \\
\hline M4 & 3270 & 1.665 & 4.266 & -0.378 \\
\hline M5 & 3125 & 1.771 & 4.722 & -0.515 \\
\hline M6 & 2990 & 1.771 & 5.143 & -0.762 \\
\hline M7 & 2880 & 1.734 & 5.513 & -1.050 \\
\hline
\end{tabular}

\subsubsection{Color Calibration}

As required by our method, we need to assume an isochrone that defines the intrinsic, photospheric, colors as a function of $T_{\text {eff }}$, for the ONC sources in our 2CD. As shown by Da Rio et al. (2010), current atmosphere models are still unable to lead to accurate predictions of optical colors for young $(\sim 2 \mathrm{Myr})$ cool stars, and empirical calibrations of photospheric colors have not been specifically carried out for young PMS stars. Also, in that work the authors show that, for the same $T_{\text {eff }}$, the photospheric colors of $\sim 2$ Myr PMS sources are not in agreement with those of MS dwarfs. Thus, we cannot either rely on calibrations of colors valid for more evolved populations. This problem therefore requires a particular care. We explain in Appendix B the procedure used to obtain the correct isochrone calibration for our $2 \mathrm{CD}$, and we report in Table 1 the colors of the isochrone. It could be argued that the assumption of a single isochrone in our $2 \mathrm{CD}$, given a possible age spread in Orion, may lead to false predictions. This is not the case, for two reasons: (1) the $2 \mathrm{CD}$ is, by its nature, independent of stellar luminosities or radii; therefore, age variations for the same $T_{\text {eff }}$ can result in differences in the photospheric colors only due to changes in the stellar surface gravity; (2) in the optical, the difference in colors between PMS and MS stars can be of up to several tenths of a magnitude (Da Rio et al. 2010). This offset, scaled down to the modest age spread within the ONC (Reggiani et al. 2011), results in negligible differences in the colors versus $T_{\text {eff }}$ relations.

In Figure 1, we present our calibrated isochrone, together with the observed sources. If ongoing mass accretion did not alter our observed colors, the sources would have been located on the model line, with only a spread given by extinction. Instead, a number of ONC members are displaced toward bluer colors, as expected by the presence of accretion flux excess. 
For our subsequent analysis, and specifically for the derivation of bolometric stellar luminosities, we also require the bolometric corrections (BC) in the $I$ band as a function of $T_{\text {eff }}$. These are defined as the difference between the bolometric magnitude of a star and the apparent $I$-band magnitude

$$
\begin{aligned}
B C & =M_{\mathrm{bol}}-M_{\lambda} \\
& =2.5 \log \left[\frac{\int f_{\lambda}(T) \cdot S_{\lambda} d \lambda}{\int f_{\lambda}(T) \cdot d \lambda} / \frac{\int f_{\lambda}(\odot) \cdot S_{\lambda} d \lambda}{\int f_{\lambda}(\odot) \cdot d \lambda}\right] .
\end{aligned}
$$

We computed the BCs by performing synthetic photometry on the spectra of Allard et al. (2011), and corrected the results based on the same offsets we determined in our calibration of the colors, to correct the I-band intrinsic magnitudes. The values of this empirical calibrated $\mathrm{BC}$ for various spectral types are also shown in Table 1 . The $\mathrm{BC}_{I}$ varies between $\sim 0.03$ and $\sim-0.70 \mathrm{mag}$ in the range of temperatures of our sources $\left(3000 \mathrm{~K}<T_{\text {eff }}<8000 \mathrm{~K}\right)$.

\subsubsection{Accretion Spectrum}

Spectra of the flux excess produced by accretion have been modeled by Calvet \& Gullbring (1998, hereafter CG98) using a hydrodynamic treatment for the infalling material and heated photosphere. It is possible, however, to reproduce a typical accretion spectrum using simpler recipes, for instance by modeling the radiative transfer within a slab of dense gas (Valenti et al. 1993) or assuming suitable combination of an optically thin emission generated in the pre-shock region and an optically thick emission generated by the heated photosphere. In the latter case, the two components can be added with a relative fraction of about $1 / 4$ and $3 / 4$, respectively (Gullbring et al. 2000). For simplicity, we follow this latter approach; for the optically thick component, we consider an $8000 \mathrm{~K}$ black body, and for the optically thin one, we modeled the emerging spectrum from a slab of gas using version 10.00 of the Cloudy software, a photoionization code last described by Ferland et al. (1998). Specifically, we assumed a slab with density $n=10^{4} \mathrm{~cm}^{-3}$ and temperature of $\sim 20,000 \mathrm{~K}$. The particular choice of these two parameters has been selected after many trials, in order to obtain an accretion spectrum as similar as possible to that of CG98. For the far-UV part of the spectrum $(\lambda \lesssim 3000 \AA)$ we follow the results of France et al. (2011) and assume a linear decrease of flux at wavelengths shortward of the Balmer continuum.

We use our photometrical colors and produce a reddeningcorrected 2CD (Figure 2), assuming again the $A_{V}$ values of Da Rio et al. (2012). In this way, the displacements of the ONC members from the isochrone are solely due to accretion and we can investigate this aspect, neglecting the extinction contribution. In this plot sources are represented as equally normalized two-dimensional Gaussian, with widths corresponding to the individual photometric error. This facilitates our visual inspection because, in this representation, sources with small errors appear sharp and bright while sources with poorer photometry are broader and less luminous, contributing to the plot mostly through their relative number. The sources are also color-coded according to their $T_{\text {eff }}$, highlighting that the dereddened sources are generally located at bluer colors than the photospheric ones, along stripes that depend on $T_{\text {eff }}$.

In Figure 2, we also show the predicted displacement from the photospheric isochrone, obtained by adding a component of the basic accretion spectrum to the theoretical (calibrated) spectra. Specifically, increasing $L_{\text {acc }} / L_{\text {tot }}$ from 0 to 1 , the colors

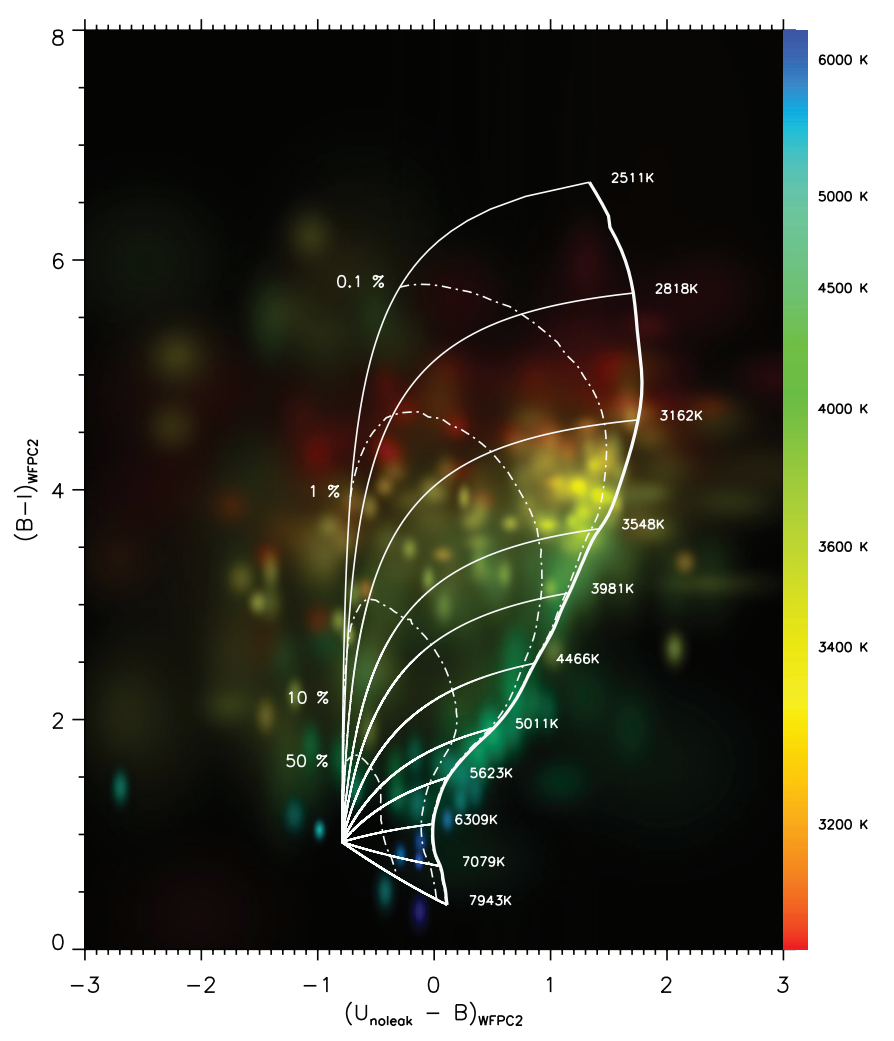

Figure 2. 2CD of our ONC population. Each source has been corrected for extinction and represented as a normalized 2D Gaussian, corresponding to the photometric errors. The sources are color-coded according to their $T_{\text {eff }}$, as shown in the scale on the right of the plot. The thick line represents our calibrated isochrone for no accretion. The thin lines represent the simulated displacements (for different $T_{\text {eff }}$ ) from the photospheric colors obtained by adding an increasing amount of $L_{\text {acc }} / L_{\text {tot }}$, assuming the accretion spectrum analogous to that of CG98. Dashed lines indicate constant ratios $L_{\mathrm{acc}} / L_{\mathrm{tot}}$.

(A color version of this figure is available in the online journal.)

are moved along lines that start from the theoretical isochrone, at any stellar $T_{\text {eff }}$, and converge to the colors of the pure accretion spectrum, located on lower left end of the plot. For this computation, we have used the basic accretion spectrum, analogous to that of CG98. It is evident that, according to this model, accretion affects both the $(B-I)$ and the $(U-B)$ colors, and this prediction is somewhat compatible with the extinctioncorrected photometry of some ONC members. On the other hand, there are many sources observed at $(U-B)<-1$, a region of this diagram which is not reachable according to this accretion spectrum model. This suggests that for these stars the Balmer jump must be higher than that of our accretion spectrum.

To correct for the discrepancy we have included another component to this spectrum in order to have a better agreement with our data. This component is modeled with an $\mathrm{H}$ II region spectrum, also simulated with Cloudy, with the same density as the one previously assumed for the optically thin part but a much lower temperature $(\sim 3000 \mathrm{~K})$. This is added to the accretion spectrum in such a way that it becomes relevant only for small amounts of mass accretion. The result is shown in Figure 3: the new model predicts, consistently with our data, a larger displacement in $(U-B)$ for low values of $L_{\text {acc }} / L_{\text {tot }}$. For our subsequent analysis, we will therefore consider this approach to model the color displacement due to mass accretion.

\subsubsection{Final Two-color Diagram}

From the modeling shown in Figure 3, we derive $L_{\text {acc }} / L_{\text {tot }}$ for all our ONC sources. For each source, we consider its known 
Table 2

Stellar Parameters of the Sources Where the 2CD Method Leads to a Determination of $A_{V}$ and $L_{\mathrm{acc}}$

\begin{tabular}{|c|c|c|c|c|c|c|c|c|}
\hline $\mathrm{OM}^{\mathrm{a}}$ & $\begin{array}{c}\text { R.A. } \\
(\mathrm{J} 2000)\end{array}$ & $\begin{array}{c}\text { Decl. } \\
(\mathrm{J} 2000)\end{array}$ & $\begin{array}{c}T_{\text {eff }}{ }^{\mathrm{b}} \\
(\mathrm{K})\end{array}$ & $\begin{array}{c}A_{V} \\
(\mathrm{mag})\end{array}$ & $\log \left(L_{*} / L_{\odot}\right)$ & $\begin{array}{c}R_{*} \\
\left(R_{\odot}\right)\end{array}$ & $\begin{array}{l}\Delta I_{\mathrm{acc}} \\
(\mathrm{mag})\end{array}$ & $\begin{array}{c}\text { Confidence }^{c} \\
\sigma\end{array}$ \\
\hline 20 & $5: 34: 15.087$ & $-5: 22: 59.971$ & 3328 & 0.56 & -1.62 & 0.47 & -0.0020 & 2 \\
\hline 30 & $5: 34: 17.299$ & $-5: 22: 47.977$ & 3104 & 1.09 & -1.51 & 0.61 & -0.0044 & 1 \\
\hline 47 & $5: 34: 20.794$ & $-5: 23: 29.131$ & 3388 & 0.51 & -1.01 & 0.90 & -0.0023 & 3 \\
\hline 50 & $5: 34: 24.769$ & $-5: 22: 10.443$ & 3465 & 0.22 & -0.82 & 1.09 & -0.0021 & 3 \\
\hline 63 & $5: 34: 26.501$ & $-5: 23: 23.986$ & 3138 & 0.95 & -0.80 & 1.35 & -0.0001 & 1 \\
\hline 69 & $5: 34: 28.962$ & $-5: 23: 48.085$ & 3107 & 0.42 & -1.61 & 0.54 & -0.0031 & 3 \\
\hline 70 & $5: 34: 29.446$ & $-5: 23: 37.437$ & 3138 & 0.08 & -1.58 & 0.55 & -0.0002 & 1 \\
\hline 77 & $5: 34: 32.658$ & $-5: 21: 7.4565$ & 3632 & 0.91 & -0.57 & 1.31 & -0.0197 & 3 \\
\hline 87 & $5: 34: 30.477$ & $-5: 21: 55.949$ & 3124 & 1.09 & -1.63 & 0.52 & -0.0056 & 1 \\
\hline 146 & $5: 34: 48.830$ & $-5: 23: 17.984$ & 3705 & 1.29 & -0.16 & 2.03 & -0.0004 & 3 \\
\hline 167 & $5: 34: 50.496$ & $-5: 23: 35.331$ & 3435 & 0.08 & -0.83 & 1.09 & -0.0364 & 3 \\
\hline$\cdots$ & $\cdots$ & $\cdots$ & $\cdots$ & $\cdots$ & $\cdots$ & $\cdots$ & $\cdots$ & \\
\hline
\end{tabular}

Notes.

a Orion Master Catalog entry number (Robberto et al. 2012).

b From Da Rio et al. (2012) and references therein.

c Sigma of confidence introduced in Section 3.1.3.

(This table is available in its entirety in a machine-readable form in the online journal. A portion is shown here for guidance regarding its form and content.)

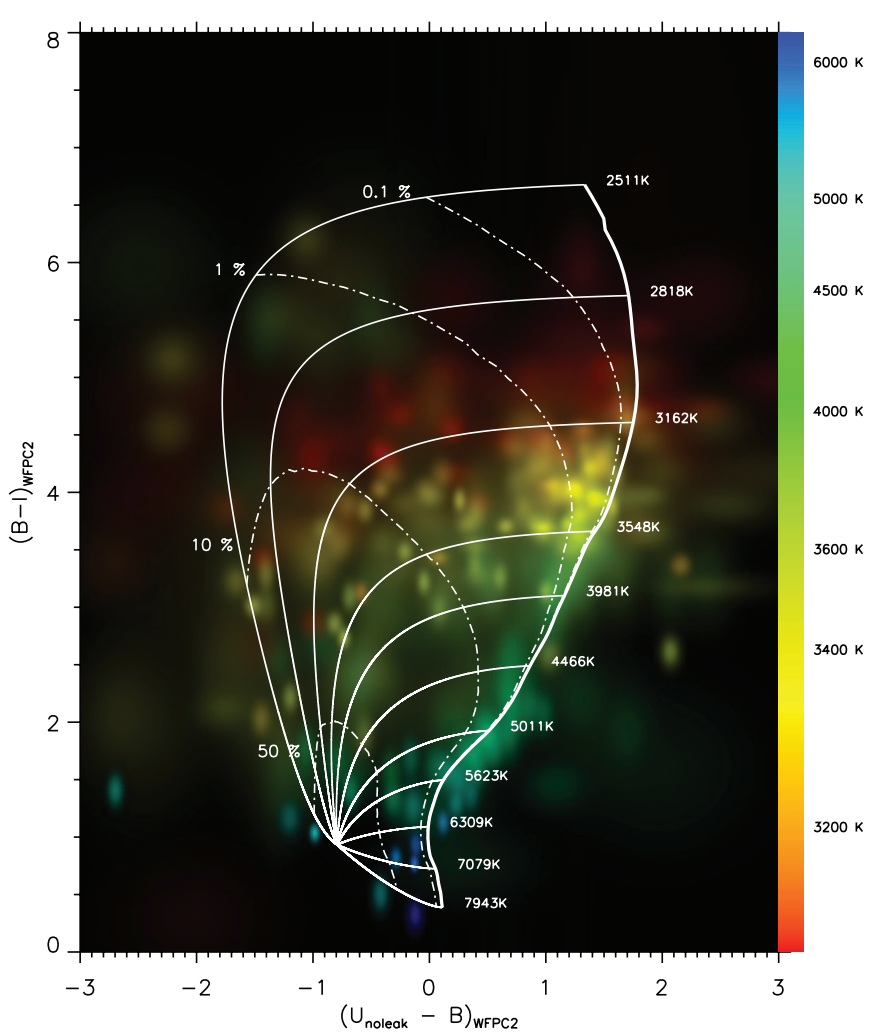

Figure 3. Same as Figure 2, but adding to the stellar spectra a sum of the accretion spectrum produced used for the model of Figure 2, and a pure $\mathrm{H}$ II region with decreasing ratio of this component to the first for increasing $L_{\text {acc}}$. (A color version of this figure is available in the online journal.)

$T_{\text {eff }}$ from Da Rio et al. (2012), and therefore the unique accretion displacement curve in the $2 \mathrm{CD}$ that starts from isochrone point corresponding to the stellar temperature of the source. We then consider the observed photometry of the source in the $2 \mathrm{CD}$ and deredden it along the reddening vector until an interception with the accretion curve is found. This intersection defines, geometrically, both the amount of $A_{V}$ and $L_{\text {acc }} / L_{\text {tot }}$ for the star.
Using this method, we obtain a solution for 271 sources. In order to quantify the uncertainty in the results, we use a Monte Carlo method: for each source we displace randomly the photometry in each band within the photometric errors, assumed Gaussian. We also randomly change the stellar $T_{\text {eff }}$ accounting for the uncertainty in the spectral types from spectroscopy, equal to \pm 1 spectral subtype (Hillenbrand 1997). We iterate this approach 1000 times for each star, deriving each time $A_{V}$ and $L_{\text {acc }} / L_{\text {tot }}$ values. From the 1000 results of $A_{V}$ and $L_{\text {acc }} / L_{\text {tot }}$ for each source, we determine the $1 \sigma, 2 \sigma$, and $3 \sigma$ intervals of the distribution of results. If an intersection is found for all the 1000 iterations, we assume our result to have a $3 \sigma$ confidence; on the other hand, if for some of the iterations an intersection is not found (because, e.g., the colors lie outside the color range reachable by the accretion model plus extinction), a lower confidence level is associated with the result. We report these values in Table 2. Neglecting sources with confidence lower than $1 \sigma$, our sample comprises 245 sources. In Figure 4, we show the distribution of $A_{V}$ and $L_{\text {acc }} / L_{\text {tot }}$ for this sample. Our extinctions vary from $\sim 0$ to $\sim 5$, with a mean value of $\sim 0.98 \pm$ 0.06 mag and only seven sources show a negative value of $A_{V}$, the lowest being $A_{V}=-0.18 \pm 0.03 \mathrm{mag}$. We assign to these sources $A_{V}=0$ and retain them in our analysis. Comparing our results with those obtained by Da Rio et al. (2012) we obtain a very good match, measuring of a distribution of differences $\Delta A_{V}=A_{V_{\text {this work }}}-A_{V_{\text {DaRio }}}$ peaked at -0.03 mag with a sigma of $0.31 \mathrm{mag}$. This testifies to the accuracy of our method.

From now on we will use these values of $A_{V}$ and $L_{\text {acc }}$ for all the sources for which this method gave a result. On the other hand, a small difference in the estimate of $L_{\text {acc }}$ with Da Rio et al. (2010) is expected, given the higher sensitivity of our 2CD to this quantity with respect to their analysis.

The Monte Carlo approach allows to estimate the errors on our parameters. We derive individual errors from the standard deviation of the values obtained; these will be used in our subsequent analysis to estimate the errors of the $\dot{M}_{\text {acc }}$.

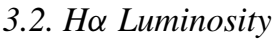

Besides the $U$-band excess, magnetospheric infall accretion processes lead to line emission, in particular the hydrogen 

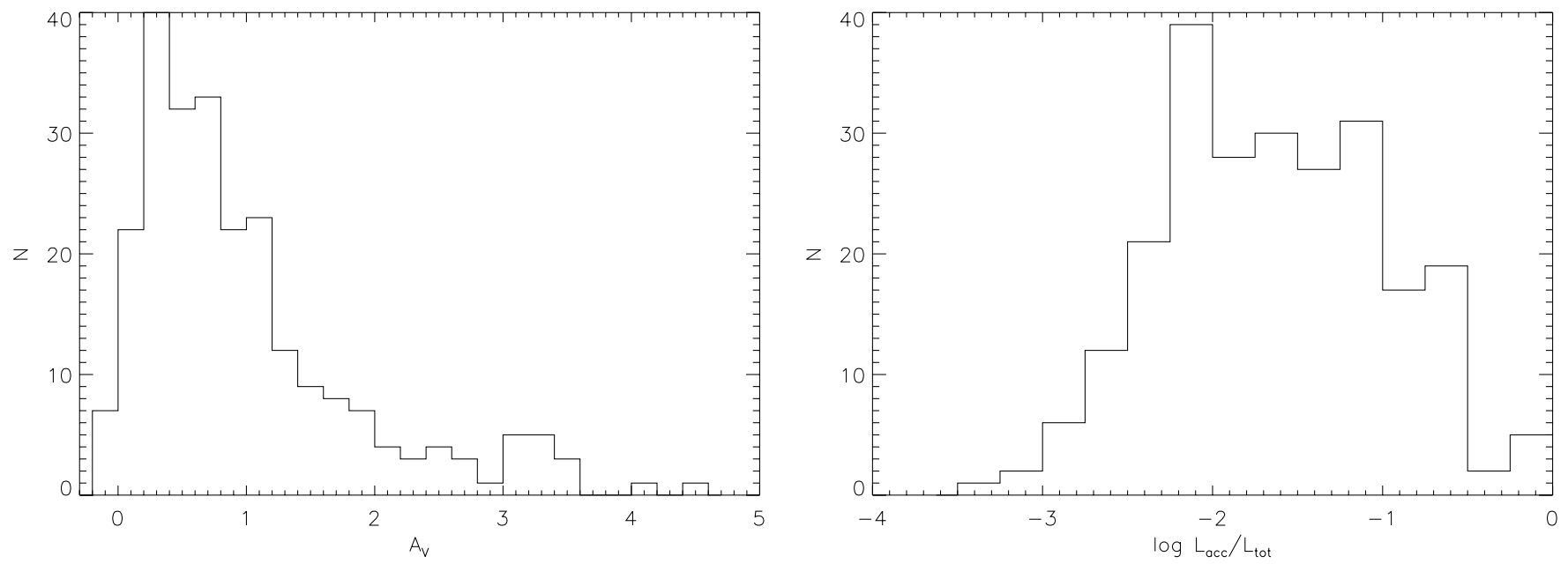

Figure 4. Histograms of the values of $A_{V}$ and $L_{\text {acc }} / L_{\text {tot }}$ estimated for our sources from the analysis of the $2 \mathrm{CD}$.

recombination lines (see, e.g., Muzerolle et al. 2001). Specifically, the $\mathrm{H} \alpha$ excess is often used as an indicator of mass accretion, and its intensity can be empirically related to $L_{\text {acc }}$.

As anticipated in Section 2.2, our WFPC2 catalog includes 1342 sources with measured $\mathrm{H} \alpha$ photometry. In order to correctly estimate the equivalent width of the $\mathrm{H} \alpha$ emission $\left(\mathrm{EW}_{\mathrm{H} \alpha}\right)$ one requires an estimate of the photospheric continuum at its wavelength. This generally depends on a nearby broadband flux (e.g., the $I$-band luminosity), as well as both $T_{\text {eff }}$ and $A_{V}$ of the sources. To this purpose, we consider temperatures from Da Rio et al. (2012) and extinctions from either our 2CD analysis (Section 3.1.3), or from Da Rio et al. (2012) for the sources with no results from the $2 \mathrm{CD}$. These required parameters reduce our sample to a total of 1026 sources. The photospheric continuum is then estimated by means of synthetic photometry, computing the photospheric $(\mathrm{H} \alpha-I)$ color for each star given its $T_{\text {eff }}$ and subtracting it from the dereddened I magnitudes. The $\mathrm{H} \alpha$ excess, measured from the difference between the observed $\mathrm{H} \alpha$ flux and the expected photospheric flux, is then converted in terms on equivalent width, or in terms of fraction of total stellar luminosities. We also stress that the WFPC2 F656N filter is broad enough $\left(\mathrm{FWHM} \sim 28.5 \AA \sim 1300 \mathrm{~km} \mathrm{~s}^{-1}\right.$ ) to contain the entire $\mathrm{H} \alpha$ excess, since this line has a typical broadening of 200-700 km s${ }^{-1}$ ( 4.5-13 А) (e.g., White \& Basri 2003; Mortier et al. 2011), much smaller than the filter width.

From all the derived values, we consider in our analysis only sources with $3<\left|\mathrm{EW}_{\mathrm{H} \alpha}\right|<1000$. This choice allows us to exclude non-accreting sources, or members showing a suspiciously high excess, probably due to uncertainties or contamination from circumstellar material. We also exclude from our analysis sources that are known from visual inspection on the HST images to be proplyds, binaries or edge-on disks, as already explained in Section 2.2. Ultimately, the final sample of sources with available $\mathrm{H} \alpha$ luminosity includes 682 ONC members. We report these results in Table 3.

\subsection{Stellar Luminosity}

In order to convert the obtained values of $L_{\mathrm{acc}} / L_{\mathrm{tot}}$, an estimate of the stellar luminosity of our sources is required. We derive the bolometric luminosity $\left(L_{*}\right)$ of each source from the observed $I$ magnitudes, corrected for the effects of extinction and accretion derived in Section 3.1.3. In particular, accretion is taken into account by computing the excess in magnitudes $\Delta I_{\text {acc }}$ in the $I$ band due to the derived amount of accretion for each source.
These values $\Delta I_{\mathrm{acc}}$ (reported in Table 2) have a mean of $\sim-0.04 \mathrm{mag}$, a rather small value, since the $I$ band is weakly affected by accretion (e.g., Fischer et al. 2011). Thus, $L_{*}$ is then derived from

$$
\begin{aligned}
\log \left(\frac{L_{*}}{L_{\odot}}\right)= & 0.4 \cdot\left[\mathbf{M}_{\mathrm{bol}, \odot}-\mathbf{M}_{\mathrm{bol}, *}\right] \\
= & 0.4 \cdot\left[\mathbf{M}_{I_{\mathrm{WFPC} 2} \odot-\mathbf{M}_{I_{\mathrm{WFPC} 2}, *}}\right. \\
& \left.+B C_{I_{\mathrm{WFPC} 2} \odot}-B C_{I_{\mathrm{WFPC} 2}, *}\right] \\
= & 0.4 \cdot\left[\mathbf{M}_{V, \odot}-\left(V-I_{\mathrm{WFPC} 2}\right)_{\odot}-I_{\mathrm{WFPC} 2}\right. \\
& \left.+\Delta I_{\mathrm{acc}}+A_{I_{\mathrm{WFPC} 2}}+B C_{I_{\mathrm{WFPC} 2}}\left(T_{\mathrm{eff}}\right)+\mathrm{DM}\right],
\end{aligned}
$$

where $I_{\mathrm{WFPC} 2}$ refers to magnitudes in the WFPC2 photometric system, and $V$ indicates the $V$-band magnitude in the standard Johnson photometry. We assume the value $M_{V, \odot}=4.83$ (Binney $\&$ Tremaine 2008), checking that using as a reference for the solar spectrum the synthetic spectrum of Allard et al. (2011) with $T_{\text {eff, } \odot}=5780 \mathrm{~K}$ and $\log g_{\odot}=4.43$ we obtain the same result, and we compute through synthetic photometry $\left(V-I_{\mathrm{WFPC} 2}\right)_{\odot}=0.70$. We assume a distance for the ONC of $d=414 \pm 7 \mathrm{pc}$ (Menten et al. 2007), corresponding to a distance modulus, $\mathrm{DM}=8.085$. Extinction is converted from $A_{V}$ to $A_{I_{\mathrm{WFPC} 2}}$ using the reddening law of Cardelli et al. (1989); also here the values of $A_{V}$ adopted are those obtained with the 2CD, if available, and from Da Rio et al. (2012), when there are no results from the $2 \mathrm{CD}$. Finally, $\mathrm{BC}_{I_{\mathrm{WFPC} 2}}\left(T_{\mathrm{eff}}\right)$ are the $I$-band BCs, derived in Section 3.1.1. The derived $L_{*}$ are reported in Table 2 in the cases where $A_{V}$ is estimated with the $2 \mathrm{CD}$ and in Table 3 in the others.

Errors in $L_{*}$ are also derived with a Monte Carlo procedure, similarly to Section 3.1 .3 , propagating the estimated uncertainties of the photometry, extinction, DM, and $\Delta I_{\text {acc }}$.

\section{RESULTS: STELLAR PROPERTIES}

\subsection{Accretion Luminosity}

\subsection{1. $L_{\text {acc }}$ from the Two-color Diagram}

We convert the derived $L_{\text {acc }} / L_{\text {tot }}$ from the $2 \mathrm{CD}$ (Section 3.1.3) in terms of $L_{\text {acc }} / L_{\odot}$. This is achieved considering the stellar luminosities $L_{*}$ derived in Section 3.3, from the relation:

$$
\frac{L_{\mathrm{acc}}}{L_{\odot}}=\frac{L_{\mathrm{acc}} / L_{\mathrm{tot}}}{1-\left(L_{\mathrm{acc}} / L_{\mathrm{tot}}\right)} \cdot \frac{L_{*}}{L_{\odot}} .
$$


Table 3

Stellar Parameters of the Sources Where We Assumed $A_{V}$ from Da Rio et al. (2012) and We Estimate $L_{\text {acc }}$ from the H $\alpha$ Luminosity

\begin{tabular}{|c|c|c|c|c|c|c|c|c|}
\hline $\mathrm{OM}^{\mathrm{a}}$ & $\begin{array}{c}\text { R.A. } \\
\text { (J2000) }\end{array}$ & $\begin{array}{c}\text { Decl. } \\
(\mathrm{J} 2000)\end{array}$ & $\begin{array}{c}T_{\mathrm{eff}}^{\mathrm{b}} \\
(\mathrm{K})\end{array}$ & $\begin{array}{c}A_{V}^{\mathrm{b}} \\
(\mathrm{mag})\end{array}$ & $\log \left(L_{*} / L_{\odot}\right)$ & $\begin{array}{c}R_{*} \\
\left(R_{\odot}\right)\end{array}$ & $\begin{array}{c}\mathrm{EW}_{\mathrm{H} \alpha} \\
(\AA)\end{array}$ & $\log \left(L_{\mathrm{H} \alpha} / L_{\odot}\right)$ \\
\hline 80 & $5: 34: 33.569$ & $-5: 22: 8.7996$ & 3165 & 1.32 & -0.80 & 1.33 & 110.40 & -3.40 \\
\hline 101 & $5: 34: 40.859$ & $-5: 22: 42.345$ & 4060 & 1.15 & 0.25 & 2.71 & 80.72 & -2.03 \\
\hline 104 & $5: 34: 41.952$ & $-5: 21: 32.102$ & 3079 & 1.24 & -1.13 & 0.96 & 31.52 & -4.33 \\
\hline 108 & $5: 34: 41.614$ & $-5: 23: 57.485$ & 3106 & 0.80 & -0.86 & 1.29 & 6.83 & -4.71 \\
\hline 111 & $5: 34: 42.477$ & $-5: 22: 46.243$ & 3059 & 1.54 & -1.13 & 0.97 & 70.33 & -4.00 \\
\hline 117 & $5: 34: 41.816$ & $-5: 21: 49.500$ & 2930 & 0.49 & -1.23 & 0.95 & 8.79 & -5.12 \\
\hline 130 & $5: 34: 44.797$ & $-5: 22: 38.543$ & 2954 & 0.83 & -1.70 & 0.54 & 53.61 & -4.79 \\
\hline 133 & $5: 34: 46.783$ & $-5: 21: 29.147$ & 3495 & 0.88 & -0.88 & 0.99 & 26.31 & -3.92 \\
\hline 134 & $5: 34: 45.953$ & $-5: 22: 50.690$ & 3052 & 2.58 & -1.11 & 0.99 & 11.37 & -4.78 \\
\hline 135 & $5: 34: 45.987$ & $-5: 22: 47.540$ & 3034 & 2.60 & -1.33 & 0.79 & 232.10 & -3.71 \\
\hline 144 & $5: 34: 46.559$ & $-5: 23: 25.585$ & 3560 & 3.40 & -0.39 & 1.67 & 92.06 & -2.85 \\
\hline$\cdots$ & $\ldots$ & $\ldots$ & $\cdots$ & $\cdots$ & $\cdots$ & $\cdots$ & $\cdots$ & $\cdots$ \\
\hline
\end{tabular}

Notes.

a Orion Master Catalog entry number (Robberto et al. 2012).

${ }^{\mathrm{b}}$ From Da Rio et al. (2012), and references therein.

(This table is available in its entirety in a machine-readable form in the online journal. A portion is shown here for guidance regarding its form and content.)

Table 4

Accretion Values of the Sources Where the 2CD Method Leads to a Determination of $A_{V}$ and $L_{\text {acc }}$, According to Different Evolutionary Models

\begin{tabular}{|c|c|c|c|c|c|c|c|c|c|c|}
\hline $\mathrm{OM}^{\mathrm{a}}$ & $\log \left(L_{\mathrm{acc}} / L_{\odot}\right)$ & $\begin{array}{c}M_{*}^{\mathrm{b}} \\
\left(M_{\odot}\right)\end{array}$ & $\begin{array}{c}\log \mathrm{Age}^{\mathrm{b}} \\
(\mathrm{yr})\end{array}$ & $\begin{array}{c}\log \dot{M}_{\mathrm{acc}}{ }^{\mathrm{b}} \\
\left(M_{\odot} \mathrm{yr}^{-1}\right)\end{array}$ & $\begin{array}{c}M_{*}{ }^{\mathrm{c}} \\
\left(M_{\odot}\right)\end{array}$ & $\begin{array}{c}\log \mathrm{Age}^{\mathrm{c}} \\
(\mathrm{yr})\end{array}$ & $\begin{array}{c}\log \dot{M}_{\mathrm{acc}}^{\mathrm{c}} \\
\left(M_{\odot} \mathrm{yr}^{-1}\right)\end{array}$ & $\begin{array}{c}M_{*}^{\mathrm{d}} \\
\left(M_{\odot}\right)\end{array}$ & $\begin{array}{c}\log \text { Age }^{\mathrm{d}} \\
(\mathrm{yr})\end{array}$ & $\begin{array}{l}\log \dot{M}_{\mathrm{acc}}{ }^{\mathrm{d}} \\
\left(M_{\odot} \mathrm{yr}^{-1}\right)\end{array}$ \\
\hline 20 & -3.26 & 0.25 & 7.4 & -10.40 & 0.20 & 7.4 & -10.30 & 0.23 & 7.4 & -10.36 \\
\hline 30 & -2.95 & 0.17 & 6.9 & -9.79 & 0.12 & 6.8 & -9.66 & 0.13 & 6.7 & -9.69 \\
\hline 47 & -2.60 & 0.25 & 6.5 & -9.45 & 0.28 & 6.8 & -9.49 & 0.25 & 6.5 & -9.45 \\
\hline 50 & -2.42 & 0.29 & 6.4 & -9.25 & 0.33 & 6.6 & -9.31 & 0.31 & 6.4 & -9.28 \\
\hline 63 & -3.59 & 0.16 & 6.1 & -10.07 & 0.19 & 6.4 & -10.15 & 0.14 & 5.9 & -10.00 \\
\hline 69 & -3.17 & 0.17 & 7.0 & -10.06 & 0.13 & 6.9 & -9.94 & 0.14 & 6.9 & -9.98 \\
\hline 70 & -4.13 & 0.18 & 7.0 & -11.04 & 0.14 & 7.0 & -10.94 & 0.14 & 6.8 & -10.92 \\
\hline 77 & -1.45 & 0.40 & 6.3 & -8.33 & 0.42 & 6.5 & -8.36 & 0.43 & 6.4 & -8.36 \\
\hline 87 & -2.99 & 0.17 & 7.1 & -9.91 & 0.13 & 7.0 & -9.77 & 0.14 & 6.9 & -9.83 \\
\hline 146 & -2.31 & 0.32 & 5.7 & -8.92 & 0.47 & 6.1 & -9.08 & 0.47 & 6.0 & -9.09 \\
\hline 167 & -1.56 & 0.27 & 6.4 & -8.37 & 0.31 & 6.6 & -8.42 & 0.28 & 6.4 & -8.38 \\
\hline$\cdots$ & $\cdots$ & $\cdots$ & $\cdots$ & $\cdots$ & $\cdots$ & $\cdots$ & $\cdots$ & $\cdots$ & $\cdots$ & $\cdots$ \\
\hline
\end{tabular}

Notes.

${ }^{\text {a } O r i o n ~ M a s t e r ~ C a t a l o g ~ e n t r y ~ n u m b e r ~(R o b b e r t o ~ e t ~ a l . ~ 2012) . ~}$

b Obtained with D’Antona \& Mazzitelli (1994) models.

c Obtained with Siess et al. (2000) models.

${ }^{\mathrm{d}}$ Obtained with Palla \& Stahler (1999) models.

(This table is available in its entirety in a machine-readable form in the online journal. A portion is shown here for guidance regarding its form and content.)

In Table 4, we report these values for a total sample of 245 sources.

$$
\text { 4.1.2. } L_{\text {acc }} \text { from } L_{\mathrm{H} \alpha}
$$

There are several empirical relations between the $L_{\mathrm{H} \alpha}$ and the total accretion luminosity. Since, however, we have an independent estimate of the $L_{\text {acc }}$ for some of our ONC sources from the $2 \mathrm{CD}$, we rederive this transformation based on our data. To this purpose, we isolate the sources where both $L_{\text {acc }}$ obtained with the 2CD and $L_{\mathrm{H} \alpha}$ are available. In particular, limiting to sources with at least a $2 \sigma$ confidence (see Section 3.1.3), we isolate a sample of 148 stars. Figure 5 shows the relation between these two quantities; to account for the overall uncertainties, including the intrinsic scatter in the values in the $\mathrm{H} \alpha$ flux due to variability (e.g., Murphy et al. 2011), we add quadratically to all the estimated errors on $\log \left(L_{\text {acc }} / L_{\odot}\right)$ a minimum value of 0.2 , and use these combined errors for the linear regression. Our fit provides the following relation:

$$
\log \left(\frac{L_{\mathrm{acc}}}{L_{\odot}}\right)=(1.31 \pm 0.03) \cdot \log \left(\frac{L_{\mathrm{H} \alpha}}{L_{\odot}}\right)+(2.63 \pm 0.13) .
$$

Herczeg \& Hillenbrand (2008), fitting the Balmer Jump and veiling features and correlating those estimated for $L_{\mathrm{acc}}$ to the spectroscopical estimated $\mathrm{EW}_{\mathrm{H} \alpha}$, found a relation with similar slope (1.20 \pm 0.16$)$. Dahm (2008) measured a slope of $(1.18 \pm 0.26)$ using 14 members of the Taurus-Auriga complex, combining the previous $L_{\text {acc }}$ estimate with their spectroscopical $\mathrm{EW}_{\mathrm{H} \alpha}$. De Marchi et al. (2010) assumed a unitary slope, implying the proportionality between $L_{\mathrm{H} \alpha}$ and $L_{\mathrm{acc}}$, and derived another relation using the same data of Dahm. We rely on our estimate, and we thus use the relation in Equation (5) to obtain $L_{\text {acc }}$ for 528 additional sources with no estimate of this quantity from the $2 \mathrm{CD}$. 


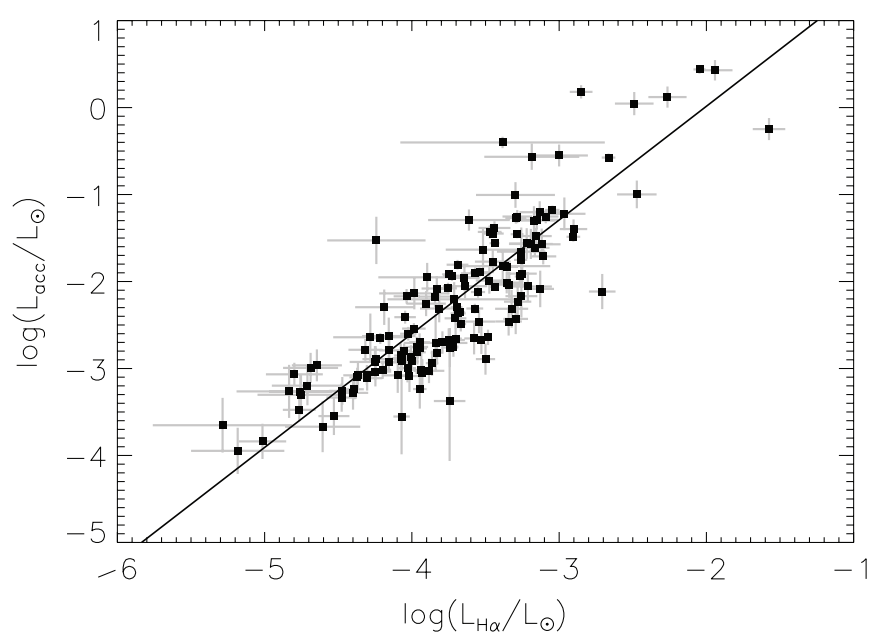

Figure 5. Calibration of the $\mathrm{H} \alpha$ luminosity as an accretion indicator using the accretion luminosity $\left(L_{\mathrm{acc}}\right)$ obtained with the $2 \mathrm{CD}$. The linear regression fit shown in figure has an analytic expression reported in the text in Equation (5) that permits to obtain accretion luminosity from the $\mathrm{H} \alpha$ luminosity estimate.

\subsection{Stellar Masses and Ages}

To derive the $\dot{M}_{\text {acc }}$ for our sources, we still need to estimate the masses of our sources $\left(M_{*}\right)$; this is accomplished using evolutionary model interpolation of the position of our sources on the Hertzprung-Russel diagram (HRD). Moreover, this procedure gives us the age of the sources that will be used in the later analysis.

In Figure 6, we present the HRD of our sources, built using $T_{\text {eff }}$ from Da Rio et al. (2012) and the luminosity $\left(L_{*}\right)$ derived in Section 3.3. Sources with a measurement of accretion from the $2 \mathrm{CD}$ are plotted in triangles, whereas accretion estimates from the $\mathrm{H} \alpha$ excess are represented by open squares. From Figure 6, it is evident that our sample of $M_{\text {acc }}$ estimates extends well down to the hydrogen-burning limit. In the very low mass range, our sample includes members at all luminosities (or ages), in agreement with the overall luminosity spread measured in Orion (Da Rio et al. 2010, 2012). We find some sources located on our HRD below the main sequence; these are either relatively less luminous sources seen in scattered light (Skemer et al. 2011) or non-members of the ONC, and will be excluded from our analysis.

We assign masses and ages to our sources by interpolating theoretical isochrones and evolutionary tracks on the HRD. We use models of D'Antona \& Mazzitelli (1994), Siess et al. (2000), and Palla \& Stahler (1999; see Figure 6). In Figure 7, we present the age and mass distributions obtained from the different evolutionary models.

Considering only sources that appear on the HRD at higher $L_{*}$ than the ZAMS, our stellar sample includes 730 sources with $M_{*}$ and age estimates from D'Antona \& Mazzitelli, 675 using Palla \& Stahler models, and 697 assuming isochrones from Siess et al.

\subsubsection{Completeness Analysis}

The completeness of these samples depends on two factors: the availability of $T_{\text {eff }}$ from Da Rio et al. (2012) and the detection of the sources in $U$ or $\mathrm{H} \alpha$ (within the WFPC2 field of view). Whereas the $U$-band photometry tends to be shallow for highly reddened stars, the $\mathrm{H} \alpha$ photometry is much less affected by extinction, and extends well into the $\mathrm{BD}$ mass range. Therefore, source detection is not expected to alter the represen-
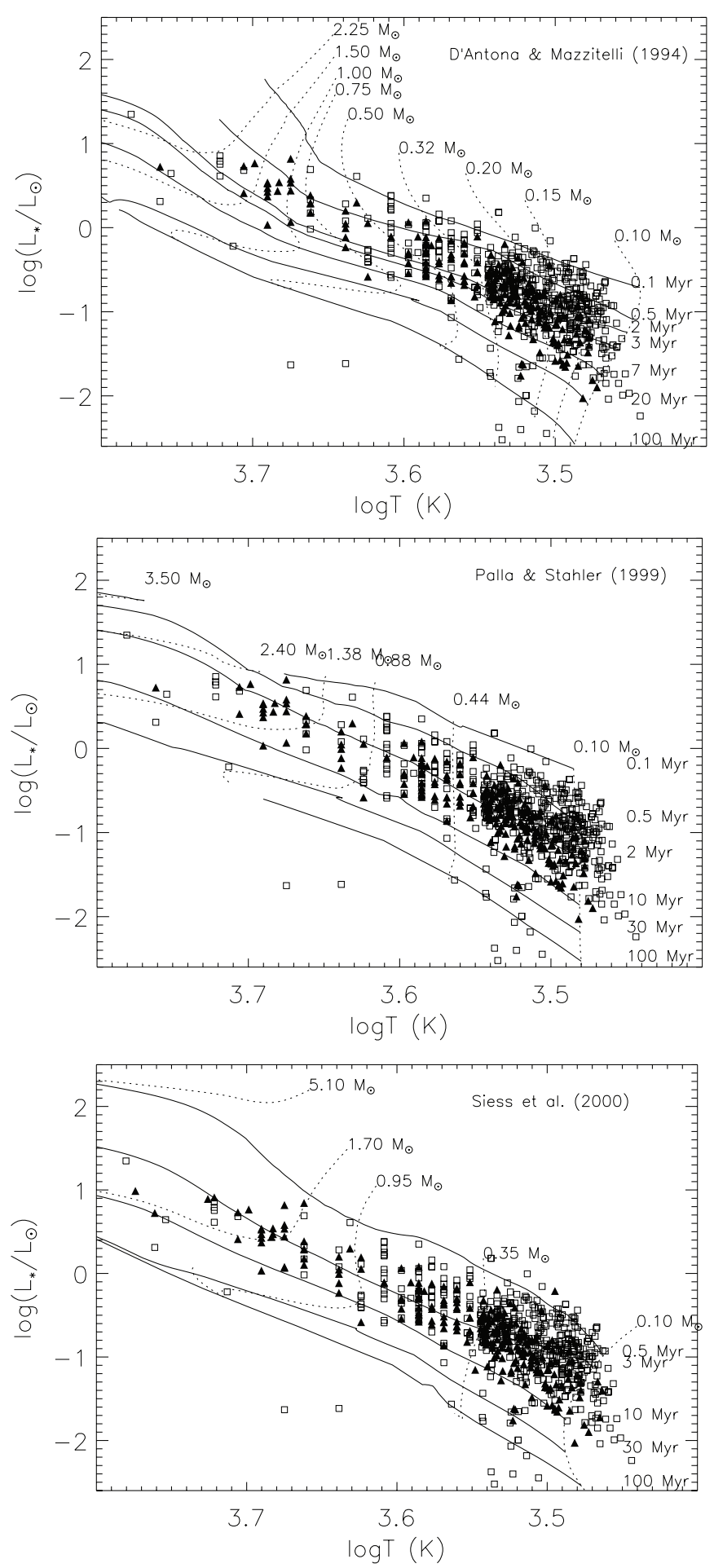

Figure 6. Hertzsprung-Russel diagram of our ONC sources with overplotted different PMS evolutionary models. Filled triangles refer to sources whose $L_{\text {acc }}$ has been estimated using the $2 \mathrm{CD}$, while empty squares are sources with results derived from the $L_{\mathrm{H} \alpha}$.

tativeness of our stellar sample. On the other hand, the available $T_{\text {eff }}$ spectral types from Da Rio et al. (2012) are characterized by a uniform and close to $100 \%$ completeness down to the H-burning limit. Therefore, we expect our sample of $\dot{M}_{\text {acc }}$ to be representative of the ONC stellar population down to $\sim 0.1 M_{\odot}$, and the lower number of sources in our catalog compared to Da Rio et al. (2012) is mainly due to our smaller field of view. To test this hypothesis, and rule out significant selection effects in 

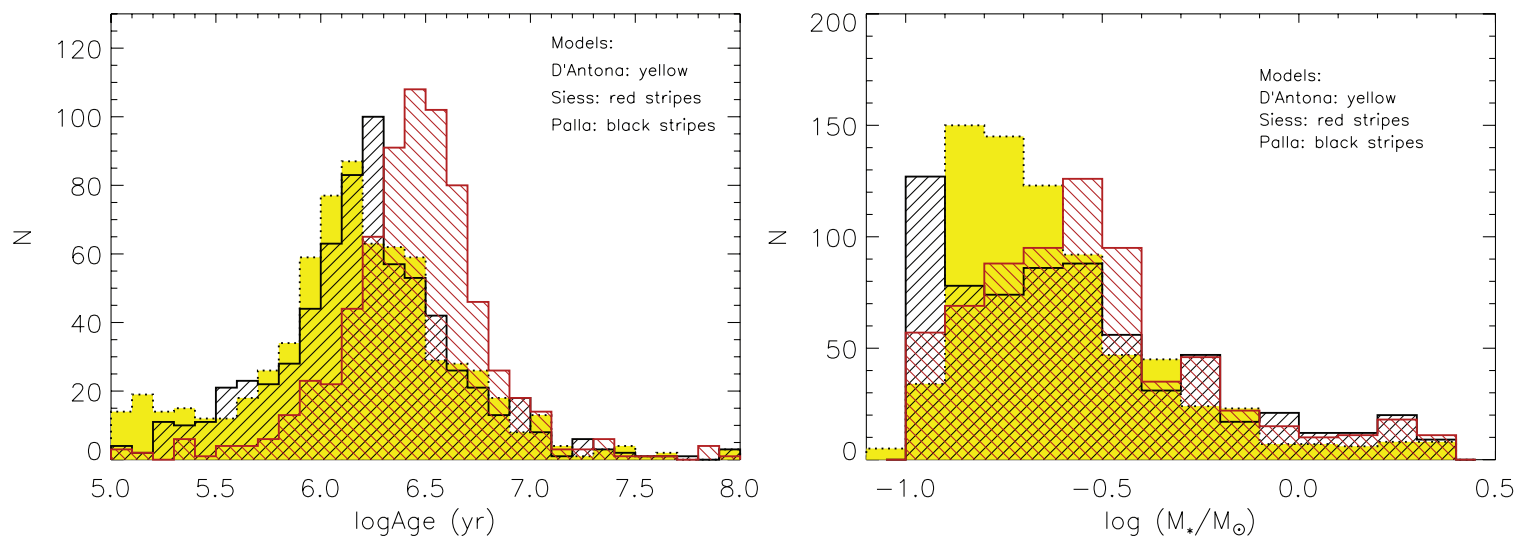

Figure 7. Age (left panel) and mass (right panel) distributions for our stellar sample, according to the three family of evolutionary models we have considered.

(A color version of this figure is available in the online journal.)
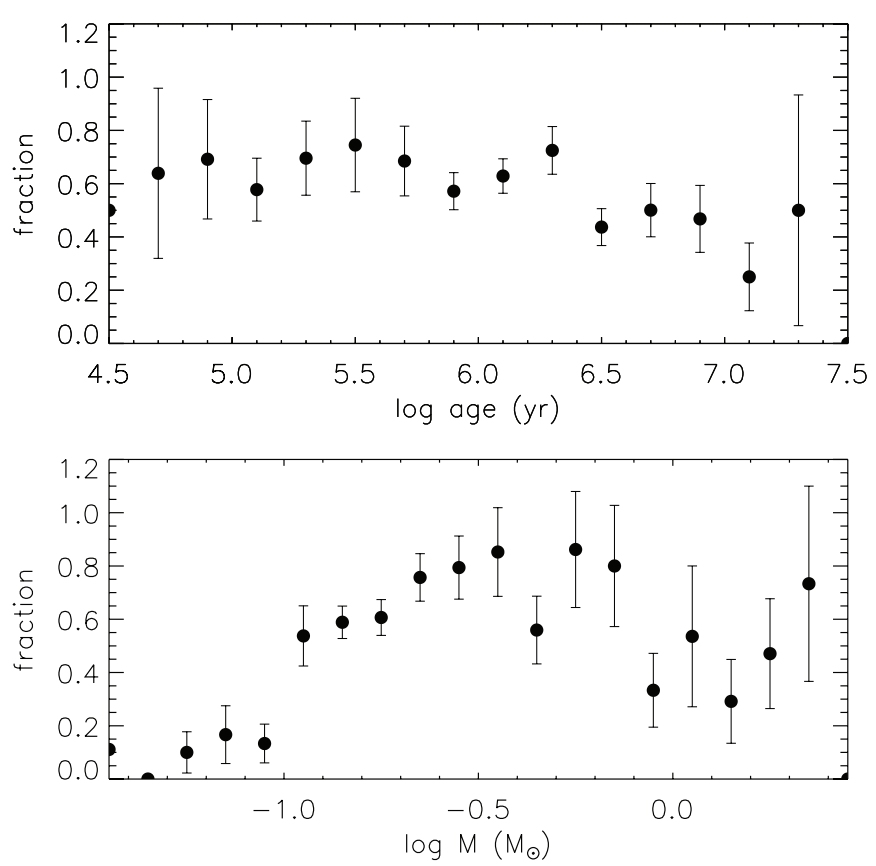

Figure 8. Fraction of the sources in our samples with respect to the sample of Da Rio et al. (2012), that is complete at $100 \%$ down to the H-burning limit. We see that we have a $\sim 70 \%$ of completeness down to the H-burning limit.

our sample of $\dot{M}_{\text {acc }}$ estimates, we consider the full catalog of available stellar parameters from Da Rio et al. (2012), limited to the same (smaller) field of view of our WFPC2 survey. Then we compute, as a function of mass and age, the fraction of these sources included in our $\dot{M}_{\text {acc }}$ sample. The result, obtained assuming D'Antona \& Mazzitelli models, is shown in Figure 8. The error bars represent the Poissonian uncertainty from the stellar numbers in both samples. As supposed, we do not detect significant trends in the fraction of accreting sources with respect to the stellar paramters, except for a significant lack of sources below $0.1 M_{\odot}$. Above this mass, about two-thirds of the ONC members have an $\dot{M}_{\text {acc }}$ measurement, regardless their mass or age.

\subsection{Mass Accretion Rates}

As shown in Equation (1), $\dot{M}_{\text {acc }}$ is derived from the measured total accretion luminosity $L_{\text {acc }}$, the stellar radius $R_{*}$, and the mass $M_{*}$. We obtain the radius of the sources from their $T_{\text {eff }}$ and $L_{*}$. The obtained values of $\dot{M}_{\text {acc }}$ are reported in Tables 4 and 5, separately for source with $L_{\text {acc }}$ estimated from the $2 \mathrm{CD}$ and from the $\mathrm{H} \alpha$ excess.

Figure 9 shows the distribution of $\dot{M}_{\text {acc }}$ according to the three evolutionary models. The distributions appear similar to each other, spanning a range between $\sim 10^{-11}$ and $\sim 10^{-7} M_{\odot} \mathrm{yr}^{-1}$, with a mean value of about $\sim 1.4 \times 10^{-9} M_{\odot} \mathrm{yr}^{-1}$. This is compatible with what was previously found for the Trapezium region (Robberto et al. 2004).

In the same plot, we also compare the distributions separately for very low mass stars $\left(M_{*}<0.3 M_{\odot}\right.$, blue dotted histograms $)$ and low- and intermediate-mass stars $\left(M_{*}>0.3 M_{\odot}\right.$, red dot-dashed histograms). It is evident that, regardless of the evolutionary model, intermediate-mass stars show higher mass accretion rates than lower mass stars. In the next section, we will investigate this trend in more detail.

\section{4. $L_{a c c}$ from Censored Data}

Our WFPC2 catalog of ONC sources provides photometric upper limits (Robberto et al. 2012) for sources undetected in one or more photometric bands; this allows us to obtain, for some sources, additional upper values of $L_{\text {acc }}$. For 55 sources with available $T_{\text {eff }}$ and detection in $B$ and $I$, but undetected in $U$ band, we could derive upper limits on $L_{\text {acc }}$ with the $2 \mathrm{CD}$ method. For eight of these stars with no correspondent $L_{\mathrm{H} \alpha}$ estimate, we find an intersection in the $2 \mathrm{CD}$, leading to an upper limit on their $L_{\text {acc }}$ that can be used in our analysis. These values are also reported in Table 4. For the other 21 of these sources with intersection on the $2 \mathrm{CD}, L_{\mathrm{acc}}$ was already derived from the $L_{\mathrm{H} \alpha}$ excess; therefore, as a sanity check, we compare these values with the upper limits obtained from $U$ : in 14 cases (66\%) we find that the upper limit value is higher than the derived one, while in the remaining $7(33 \%)$ cases the two results are compatible.

For 84 sources we have $\mathrm{H} \alpha$-band upper limit values and corresponding spectral types, therefore we are able to derive the upper limit of $L_{\mathrm{acc}}$ from Equation (5). These values are reported in Table 5.

\section{ANALYSIS: DEPENDENCE OF $L_{\text {acc }}$ AND $\dot{M}_{\text {acc }}$ ON STELLAR PARAMETERS}

$$
\text { 5.1. } L_{\text {acc }} \text { versus } L_{*}
$$

In Figure 10, we plot $L_{\text {acc }} / L_{\odot}$ as a function of $L_{*} / L_{\odot}$. We stress that these two quantities are independent of the assumed evolutionary model, allowing a less biased analysis of the dependence of accretion with the stellar properties. Because 
Table 5

Accretion Values of the Sources Where We Assumed $A_{V}$ from Da Rio et al. (2012) and We Estimate $L_{\text {acc }}$ from the H $\alpha$ Luminosity, According to Different Evolutionary Models

\begin{tabular}{|c|c|c|c|c|c|c|c|c|c|c|}
\hline $\mathrm{OM}^{\mathrm{a}}$ & $\log \left(L_{\mathrm{acc}} / L_{\odot}\right)$ & $\begin{array}{c}M_{*}^{\mathrm{b}} \\
\left(M_{\odot}\right)\end{array}$ & $\begin{array}{c}\log \operatorname{Age}^{\mathrm{b}} \\
(\mathrm{yr})\end{array}$ & $\begin{array}{c}\log \dot{M}_{\mathrm{acc}}{ }^{\mathrm{b}} \\
\left(M_{\odot} \mathrm{yr}^{-1}\right)\end{array}$ & $\begin{array}{c}M_{*}^{\mathrm{c}} \\
\left(M_{\odot}\right)\end{array}$ & $\begin{array}{c}\log \operatorname{Age}^{c} \\
(y r)\end{array}$ & $\begin{array}{c}\log \dot{M}_{\mathrm{acc}}^{\mathrm{c}} \\
\left(M_{\odot} \mathrm{yr}^{-1}\right)\end{array}$ & $\begin{array}{c}M_{*}^{\mathrm{d}} \\
\left(M_{\odot}\right)\end{array}$ & $\begin{array}{c}\log \operatorname{Age}^{\mathrm{d}} \\
(\mathrm{yr})\end{array}$ & $\begin{array}{l}\log \dot{M}_{\mathrm{acc}}{ }^{\mathrm{d}} \\
\left(M_{\odot} \mathrm{yr}^{-1}\right)\end{array}$ \\
\hline 80 & -1.82 & 0.17 & 6.1 & -8.33 & 0.21 & 6.4 & -8.42 & 0.16 & 6.0 & -8.29 \\
\hline 101 & -0.03 & 0.40 & 5.4 & -6.60 & 0.75 & 5.9 & -6.87 & 0.80 & 5.8 & -6.90 \\
\hline 104 & -3.04 & 0.16 & 6.3 & -9.66 & 0.15 & 6.6 & -9.63 & 0.12 & 6.2 & -9.54 \\
\hline 108 & -3.54 & 0.15 & 6.1 & -10.02 & 0.18 & 6.4 & -10.09 & 0.13 & 6.0 & -9.94 \\
\hline 111 & -2.61 & 0.15 & 6.3 & -9.21 & 0.14 & 6.6 & -9.18 & 0.11 & 6.2 & -9.07 \\
\hline 117 & -4.08 & 0.12 & 6.4 & -10.60 & 0.10 & 6.6 & -10.51 & $<0.10$ & $\ldots$ & $\ldots$ \\
\hline 130 & -3.65 & 0.11 & 6.8 & -10.34 & $<0.10$ & $\ldots$ & $\ldots$ & $<0.10$ & $\ldots$ & $\ldots$ \\
\hline 133 & -2.50 & 0.31 & 6.5 & -9.40 & 0.34 & 6.7 & -9.44 & 0.33 & 6.6 & -9.43 \\
\hline 134 & -3.64 & 0.15 & 6.3 & -10.21 & 0.14 & 6.6 & -10.17 & 0.11 & 6.2 & -10.07 \\
\hline 135 & -2.22 & 0.15 & 6.5 & -8.91 & 0.11 & 6.7 & -8.76 & 0.10 & 6.3 & -8.74 \\
\hline 144 & -1.11 & 0.31 & 5.9 & -7.78 & 0.38 & 6.2 & -7.87 & 0.37 & 6.2 & -7.86 \\
\hline$\ldots$ & $\ldots$ & $\ldots$ & $\ldots$ & $\ldots$ & $\ldots$ & $\ldots$ & $\ldots$ & $\ldots$ & $\ldots$ & $\ldots$ \\
\hline
\end{tabular}

Notes.

a Orion Master Catalog entry number (Robberto et al. 2012).

b Obtained with D'Antona \& Mazzitelli (1994) models.

c Obtained with Siess et al. (2000) models.

${ }^{\mathrm{d}}$ Obtained with Palla \& Stahler (1999) models.

(This table is available in its entirety in a machine-readable form in the online journal. A portion is shown here for guidance regarding its form and content.)

of this, such a relation has been long investigated: Clarke \& Pringle (2006), reporting results from Natta et al. (2006), showed an almost uniform distribution of sources on this plot between the upper bound $L_{\text {acc }} \sim L_{*}$ and the lower observational detection threshold, with a relation $L_{\text {acc }} \propto L_{*}^{\beta}$ with $\beta=1.6$. Tilling et al. (2008) were able to theoretically reproduce the observed dispersion for $M_{*} \geqslant 0.4 M_{\odot}$, even if they could not reach the lowest values of $L_{\text {acc }}$ detected observationally. Assuming D'Antona \& Mazzitelli evolutionary models, they used a dependence of $\dot{M}_{\text {acc }} \propto t^{-\eta}$ with $\eta=1.5$ (Hartmann et al. 1998), obtaining $\beta=1.7$.

Figure 10 shows that our plot is widely populated between $L_{\text {acc }} \sim L_{*}$ and $L_{\text {acc }} \sim 0.01 L_{*}$, with several sources below this range, down to $L_{\text {acc }} \sim 0.001 L_{*}$. The upper locus found by Natta et al. (2006) is still present at $L_{\text {acc }} \sim L_{*}$ in our sample. We observe 21 sources over this level, but 17 of which are compatible within $2 \sigma$ to $L_{\text {acc }} \leqslant L_{*}$. The remaining four sources may be "continuum stars" (Calvet \& Gullbring 1998), i.e., sources where the accretion component dominates over the stellar emission. If this were the case, their spectral types might also be highly uncertain, with also an impossibility to determine the correct $L_{*}$.

We note also that the density of sources with $L_{*} \gtrsim L_{\odot}$ is much lower than that of lower luminosity sources, probably because of observational selection effects. In particular, as will be pointed out in Section 5.2.3, for higher mass stars our 2CD does not allow us to detect low values of $L_{\text {acc}}$. Vice versa, the lack of high values of $L_{\text {acc }}$ for $L_{*} \lesssim 0.3 L_{\odot}$ occurs in a region widely populated by upper limit detections and thus cannot be attributed to observational limits.

Fitting our results with a linear least-squares procedure, accounting for the errors (corrected as in Section 3.1.3 for both stellar and accretion luminosity), we obtain a slope $\beta=1.68 \pm$ 0.02. This is also shown in Figure 10 as a solid line. This value is compatible with those obtained observationally by Natta et al. (2006) and theoretically from Tilling et al. We note, however, that the slope slightly changes when selecting only sources with
$L_{\text {acc }}$ determined from the $2 \mathrm{CD}$, for which $\beta=1.73 \pm 0.02$ (dotted line), or from $L_{\mathrm{H} \alpha}$, for which $\beta=1.59 \pm 0.04$ (dashed line).

\section{2. $\dot{M}_{\text {acc }}$ versus Age and $M_{*}$ \\ 5.2.1. Fit to the Data}

To investigate the variations of $\dot{M}_{\text {acc }}$ with respect to the stellar age and mass, we consider all of these three quantities in a single $3 \mathrm{D}$ space. To this purpose, we neglect sources with suspicious ages, specifically, too young $(\log (t / y r)<5.5)$ or too old $(\log$ $(t / \mathrm{yr})>7.3)$.

We perform a minimum $\chi_{\text {red }}^{2}$ fit, accounting for errors on the single values, assuming two different forms: (1) a simple plane $\left(\log \dot{M}_{\mathrm{acc}}=A+B \cdot \log t+C \cdot \log M_{*}\right)$ and (2) a warped surface $\left(\log \dot{M}_{\mathrm{acc}}=A+B \cdot \log t+C \cdot \log M_{*}+D \cdot \log t \cdot \log M_{*}\right)$. Specifically, the first form is the simplest function to describe correlations of $\dot{M}_{\text {acc }}$ with the stellar parameters; the second allows for a mixed term that accounts for a possible different evolution timescale of $\dot{M}_{\text {acc }}$ with stellar mass. To perform the fit we use a standard Levenberg-Marquard nonlinear regression. In the two cases the resulting $\chi_{\text {red }}^{2}$ are comparable ( 7-9), and the second one is slightly smaller. This decrease of $\chi_{\text {red }}^{2}$ is not surprising, given that the first function is a subcase of the second, the latter having an additional parameter. Therefore, in order to quantitatively decide whether the most complex model is truly more representative of the real distribution of the data, or whether the simplest model is good enough given the uncertainties, we run a statistical $F$ test. This test compares the relative increase in the residual sum of squares obtained by reducing the complexity of the model, with the relative increase of the degrees of freedom. Then, the test derives the probability that the simpler model is suitable enough. In any case, we find this probability to be lower than $0.1 \%$, meaning that introducing the fourth parameter $D$ in our model (i.e., a warped surface instead of a flat plane) leads to a more representative match with our data, and this is not a statistical casualness. Our fitted surface is shown in Figure 11 

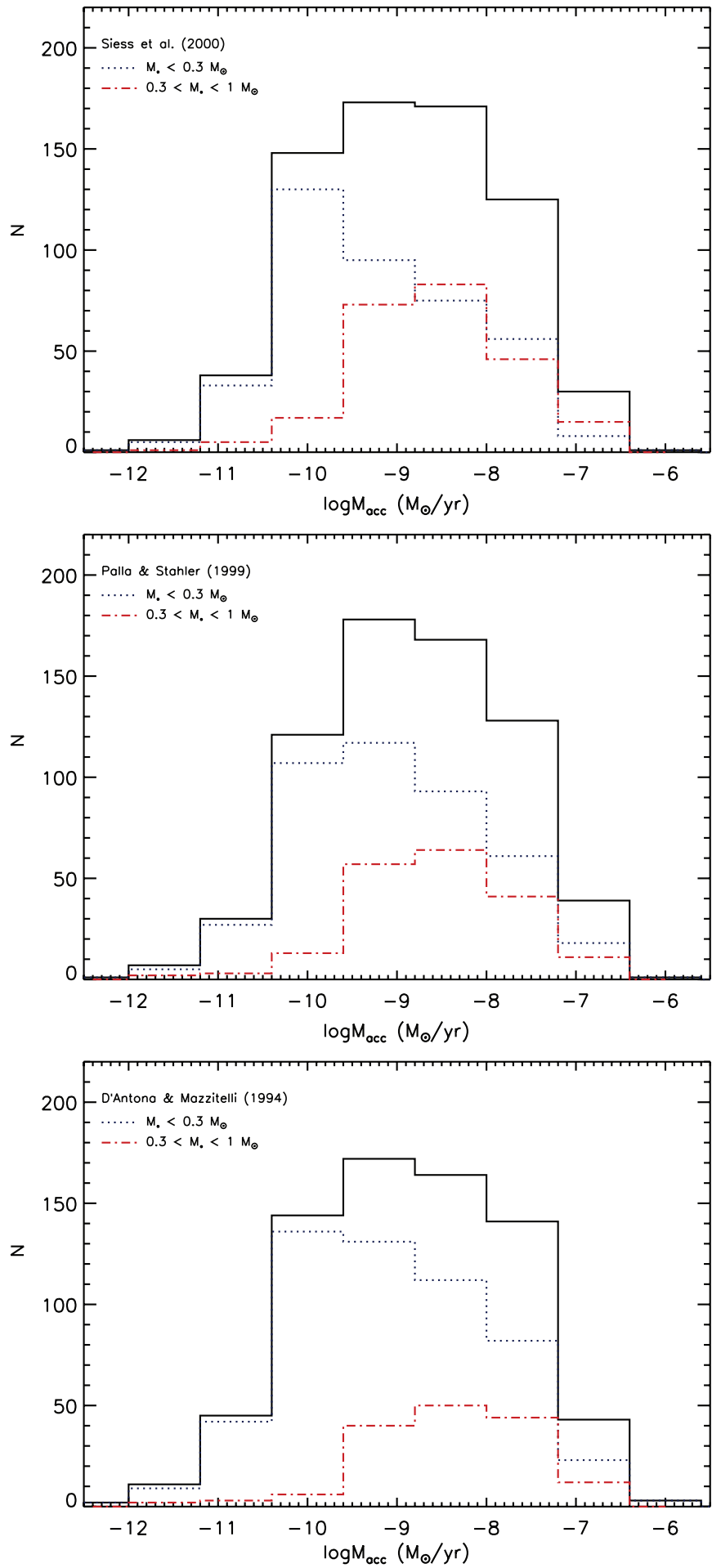

Figure 9. Distribution of mass accretion rates according to the three different evolutionary models. In each plot, we also present the partial distributions separating the sample in two mass bins, highlighting the different averages.

(A color version of this figure is available in the online journal.)

and described by the following equation:

$$
\log \dot{M}_{\mathrm{acc}}=A+B \cdot \log t+C \cdot \log M_{*}+D \cdot \log t \cdot \log M_{*},
$$

where the parameters' values are reported in Table 6 , also in the cases where we consider only sources with results obtained from the $2 \mathrm{CD}$ or with accretion estimated using the $\mathrm{H} \alpha$ luminosity.

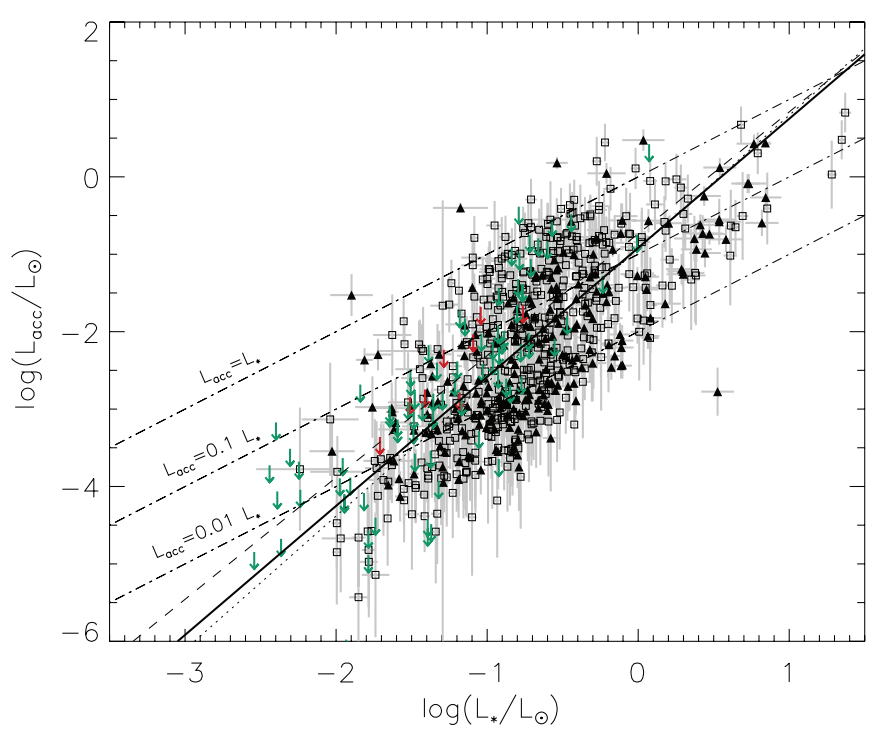

Figure 10. Accretion luminosity vs. stellar luminosity. Values obtained from the $2 \mathrm{CD}$ are plotted as triangles, while squares represent data obtained from $\mathrm{H} \alpha$ excess. Dot-dashed lines represent different ratio between accretion and stellar luminosity and the solid line is our best fit (slope $\sim 1.68$ ) from a linear least-squares regression which accounts for errors on both $L_{*}$ and $L_{\mathrm{acc}}$. The fit assuming only results from the $2 \mathrm{CD}$ is showed with a dotted line, while the one from only $\mathrm{H} \alpha$ luminosities with a dashed line.

(A color version of this figure is available in the online journal.)

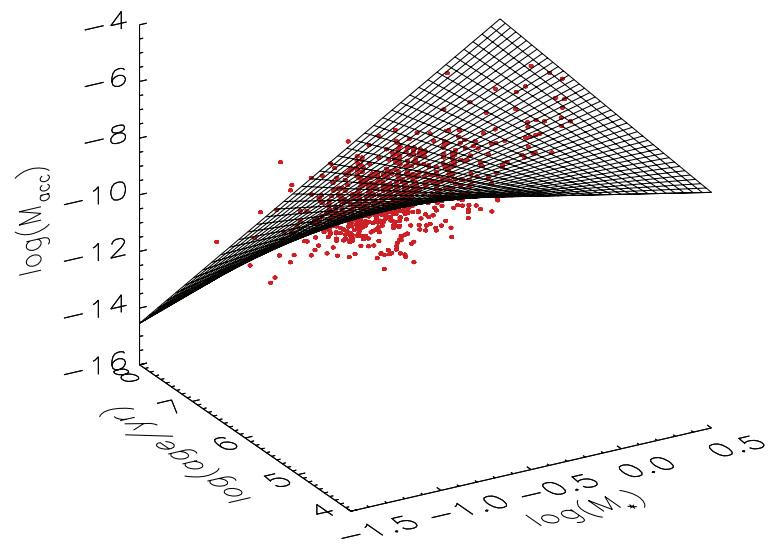

Figure 11. Snapshot of our 3D diagram of $\dot{M}_{\text {acc }}$ with respect to both $M_{*}$ and age, together with our best-fit surface (see the text). The analytical expression of the latter is also reported in Equation (6) and the coefficients of the fit are reported in Table 6 . The full 3D animation is available electronically. In Figure 12, different views of this surface are shown.

(An animation and a color version of this figure are available in the online journal.)

These relations imply that both parameters together play a role in the evolution of mass accretion. To better understand this behavior, and in particular the role of the "mixed" term described by the parameter $D$, we separately investigate the dependence of $M_{\text {acc }}$ on age or mass; also, in every case, we divide the sample in different ranges for these parameters. This is illustrated in Table 7 for the dependence on age, and in Table 8 for the dependence on mass, and the plots are shown in Figure 12.

\subsubsection{Discussion}

If we consider only the dependence of $\dot{M}_{\text {acc }}$ on stellar ages $\left(\dot{M}_{\text {acc }} \propto t^{-\eta}\right)$, our fit implies that a similar slope as that proposed by Hartmann et al. (1998) $(\eta=1.5)$ is obtained for $M_{*} \sim 0.13 M_{\odot}$. For higher mass stars we find decreasingly 


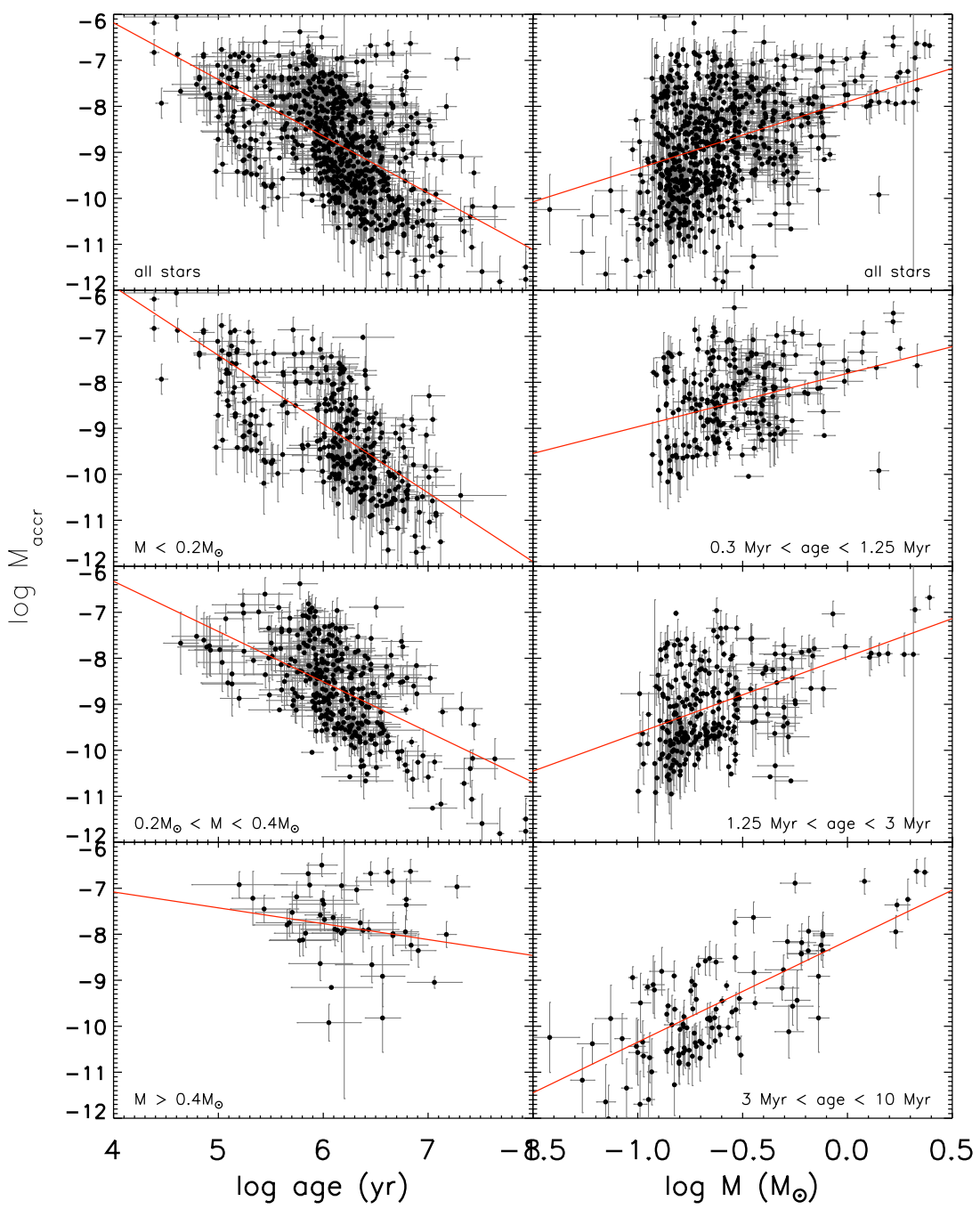

Figure 12. $\dot{M}_{\text {acc }}$ evolution as a function of individual age and mass of the sources for different ranges of these parameters. The red line is plotted according to the value of the fit obtained using all the sources in the sample (Equation (6)) and for the mean value of the parameter in the range written on the picture.

(A color version of this figure is available in the online journal.)

Table 6

Coefficients of Equation (6) Obtained Fitting the Values of $\dot{M}_{\text {acc }}$ as a Function of Both $M_{*}$ and the Age of the Sources for Different Subsamples

\begin{tabular}{lcccc}
\hline \hline Sample & $A$ & $B$ & $C$ & $D$ \\
\hline All sources & $-(5.12 \pm 0.86)$ & $-(0.46 \pm 0.13)$ & $-(5.75 \pm 1.47)$ & $(1.17 \pm 0.23)$ \\
Only 2CD results & $-(4.70 \pm 1.29)$ & $-(0.56 \pm 0.21)$ & $(0.35 \pm 0.35)$ & $-(0.54 \pm 2.20)$ \\
Only H $\alpha$ results & $-(3.29 \pm 1.36)$ & $-(0.71 \pm 0.21)$ & $(0.93 \pm 0.34)$ & $-(4.01 \pm 2.19)$ \\
\hline
\end{tabular}

Note. The sample is divided in subsamples according to the method used to obtain $L_{\text {acc }}$ and thus $\dot{M}_{\text {acc}}$.

lower values of $\eta$ with mass. Table 7, in particular, shows that the values of $\eta$ decrease monotonically regardless of considering all sources, only those with results obtained from the $2 \mathrm{CD}$ or with the $\mathrm{H} \alpha$ luminosity. Thus, our data indicate that there are different accretion evolutionary timescales for sources with different mass. Surprisingly, for sources with $M_{*} \gtrsim 0.5 M_{\odot}$ the slope is smaller than 1 . This value is not compatible with the similarity solution model framework (Lynden-Bell \& Pringle 1974; Hartmann et al. 1998). Indeed, if we suppose that the disk evolves with time following a simple power law $\left(M_{\text {disk }} \propto t^{-\alpha}\right)$, we have that $\dot{M}=d M_{d} / d t \propto t^{-(\alpha+1)}$; thus $\eta=\alpha+1>1$ in order to have a disk mass decreasing with time.
On the other hand, according to the self-similar models, $\dot{M}_{\text {acc }}$ scales with time as a simple power law only in the asymptotic regime, when the age of the system $t \gg t_{v}$, where $t_{v}$ is the viscous timescale of the disk (set by the initial conditions and by the relevant viscosity). At earlier times, the relationship between $\dot{M}_{\text {acc }}$ and $t$ is indeed shallower. Our results might thus be explained within the framework of the self-similar solutions if higher mass stars are not yet in the asymptotic regime, implying that the viscous time is a growing factor of the stellar mass. Note that this trend is the opposite of what suggested by Alexander $\&$ Armitage (2006) in the BD regime to explain the $M_{*}-\dot{M}_{\text {acc }}$ correlation. 
Table 7

$\dot{M}_{\text {acc }}$ versus Age Relation Coefficients

\begin{tabular}{lccc}
\hline \hline & $\eta$ & $\eta(2 \mathrm{CD})$ & $\eta(\mathrm{H} \alpha)$ \\
\hline$M_{*} \sim 0.13 M_{\odot}$ & $1.50 \pm 0.26$ & $0.87 \pm 0.41$ & $1.53 \pm 0.40$ \\
$M_{*} \sim 0.2 M_{\odot}$ & $1.28 \pm 0.21$ & $0.80 \pm 0.32$ & $1.36 \pm 0.32$ \\
$M_{*} \sim 0.3 M_{\odot}$ & $1.07 \pm 0.18$ & $0.74 \pm 0.28$ & $1.20 \pm 0.28$ \\
$M_{*} \sim 0.5 M_{\odot}$ & $0.81 \pm 0.15$ & $0.66 \pm 0.23$ & $0.99 \pm 0.23$ \\
$M_{*} \sim 0.8 M_{\odot}$ & $0.58 \pm 0.13$ & $0.59 \pm 0.21$ & $0.80 \pm 0.21$ \\
$M_{*} \sim 1 M_{\odot}$ & $0.46 \pm 0.13$ & $0.56 \pm 0.21$ & $0.71 \pm 0.21$ \\
\hline
\end{tabular}

Notes. Results of the fit $\dot{M}_{\text {acc }} \propto t^{-\eta}$ obtained considering all the sources (second column), sources with results obtained with the 2CD (third column), and with the $\mathrm{H} \alpha$ luminosity (fourth column). Values of the slope are different for stars belonging to subsamples with different mean $M_{*}$ (first column).

Alternatively, one might question the validity of the selfsimilar models themselves in describing, from a statistical point of view, the evolution of protostellar disks, and in particular the assumption that viscosity scales with radius as a simple power law. Indeed, different physical processes are expected to redistribute angular momentum within the disk at different radii, with the magneto-rotational instability (MRI; Balbus \& Hawley 1992) dominating at small distances from the star, the gravitational instability (Lodato \& Rice 2004) dominating in the colder outer parts, and with the possibility of having extended dead zones (Gammie 1996) in between the two.

For what concerns the dependence of $\dot{M}_{\text {acc }}$ over mass (see Table 8) modeled as $\dot{M}_{\text {acc }} \propto M_{*}^{b}$, the trend is similar regardless on the method used to estimate $\dot{M}_{\text {acc }}$. In particular, the slope in higher, thus the relation is steeper, when considering older ages. This also shows that at older ages more massive sources maintain a higher accretion rate.

Figure 12 illustrates once more these trends. We plot the evolution of our $\dot{M}_{\text {acc }}$ values against age and $M_{*}$ for different ranges of the parameters. In this figure, the slopes of the overplotted lines are obtained from Equation (6), substituting to the projected parameter (mass for the left panels, age for the right ones), the mean value within the subsample used for each particular panel.

The slower temporal decay of $\dot{M}_{\text {acc }}$ that we measure in the intermediate-mass range $\left(M_{*}>0.4 M_{\odot}\right)$ is in agreement with estimates of mass accretion rates from $\mathrm{H} \alpha$ photometry obtained in the Magellanic Clouds (De Marchi et al. 2010, 2011; Spezzi et al. 2012) and in the massive galactic cluster NGC 3603 (Beccari et al. 2010). Indeed, in all these studies, the investigated stellar samples do not reach the low-mass regime, due to poor photometric sensitivity. This suggests, as inferred by the authors of these works, that their relatively high values of $\dot{M}_{\text {acc }}$ measured for isochronal ages of $\sim 10 \mathrm{Myr}$ compared to galactic low-mass stars indicate a selection effect because of higher masses.

\subsubsection{Caveats}

Our derivation of $\dot{M}_{\text {acc }}$ using the 2CD may be, in principle, affected by some modest selection effects. This, as mentioned, is located in the fact that for high $T_{\text {eff }}$ sources, the color displacement due to accretion is very small, limiting the possibility to identify weak accretors among intermediate mass stars. Specifically, Figure 3 shows that for $(B-I) \lesssim 2$, the $1 \% L_{\text {acc }} / L_{\text {tot }}$ line is undistinguishable from the zero accretion one. This is simply because stars with high $T_{\text {eff }}$ emit a non-negligible fraction of their flux in the $U$ band, "hiding" a possible small accretion excess. As mentioned, however, this problem is highly mitigated since we have also derived $L_{\mathrm{acc}}$ from the $L_{\mathrm{H} \alpha}$ excess for all the
Table 8

$\dot{M}_{\text {acc }}$ versus $M_{*}$ Relation Coefficients

\begin{tabular}{lccc}
\hline \hline & $b$ & $b(2 \mathrm{CD})$ & $b(\mathrm{H} \alpha)$ \\
\hline Age $\sim 0.8 \mathrm{Myr}$ & $1.15 \pm 2.00$ & $1.51 \pm 3.02$ & $1.50 \pm 2.97$ \\
Age $\sim 1 \mathrm{Myr}$ & $1.26 \pm 2.02$ & $1.54 \pm 3.04$ & $1.59 \pm 2.99$ \\
Age $\sim 2 \mathrm{Myr}$ & $1.61 \pm 2.06$ & $1.65 \pm 3.12$ & $1.87 \pm 3.06$ \\
Age $\sim 5 \mathrm{Myr}$ & $2.08 \pm 2.13$ & $1.79 \pm 3.22$ & $2.24 \pm 3.16$ \\
Age $\sim 8 \mathrm{Myr}$ & $2.32 \pm 2.16$ & $1.86 \pm 3.27$ & $2.43 \pm 3.21$ \\
Age $\sim 10 \mathrm{Myr}$ & $2.43 \pm 2.18$ & $1.89 \pm 3.29$ & $2.52 \pm 3.23$
\end{tabular}

Notes. Results of the fit $\dot{M}_{\text {acc }} \propto M_{*}^{b}$ obtained considering all the sources (second column), sources with results obtained with the $2 \mathrm{CD}$ (third column) and with the $\mathrm{H} \alpha$ luminosity (fourth column). Values of the slope are different for stars belonging to subsamples with different mean age (first column).

sources for which $\dot{M}_{\text {acc }}$ is not derived from the 2CD. In the end, with the $\mathrm{H} \alpha$ measurements, and even considering upper limits (see, e.g., Figure 10) the relations we find do not appear to suffer from this mentioned selection effect.

As already introduced in Section 4.2, the accuracy of the isochronal ages to represent the true stellar ages has been recently questioned. Baraffe et al. (2009) and Baraffe \& Chabrier (2010) claimed that the observed spread of $L_{*}$ in the HRD of star forming regions can be explained as a consequence of the protostellar phase accretion history. In particular, episodic events of intense accretion $\left(\dot{M}_{\mathrm{acc}} \sim 10^{-4} M_{\odot} \mathrm{yr}^{-1}\right)$ during this phase can produce a large spread of values of stellar radii for stars of identical age and mass. Their results are based on the assumption of "cold" accretion and initial masses $M_{\mathrm{i}}$ between $1 M_{J}$ and $0.1 M_{\odot}$. On the other hand, Hosokawa et al. (2011) demonstrate that the apparent spread produced by different accretion histories may be important only for sources with $T_{\text {eff }} \gtrsim 3500 \mathrm{~K}$ ( $\sim 30 \%$ of our sources $)$. In their analysis they assume a fixed $M_{\mathrm{i}}=0.01 M_{\odot}$, and this could suggest that the results obtained should be revised changing the value of this parameter. Thus, from a theoretical point of view the debate is still ongoing and a unified picture is not yet reached. At the present moment, therefore, we can only use the age inferred from the evolutionary tracks used in the past (e.g., D'Antona \& Mazzitelli 1994), waiting for new isochrones based on episodic accretion models.

We stress that if the isochronal ages we assume are inaccurate, because of the aforementioned effects, this results in an overall "horizontal" broadening of our results in the $\dot{M}_{\text {acc }}$ versus age plane, but the overall trends we measure cannot be strongly influenced.

\section{3. $M_{\text {acc }}$ versus Position}

We look for spatial variations of $M_{\text {acc }}$ within the ONC. Such variations, in fact, might imply that the accretion is subject to environmental effects, such as the influence of the massive stars present in the central region of the cluster, in particular $\theta^{1}$ Orionis $\mathrm{C}$, an O-type star.

In order to do that, we divide our sample in five subsamples, each with the same amount of sources, according to the distance of each object from $\theta^{1} \mathrm{C}$. We show with black squared symbols in Figure 13 the mean values of the $\dot{M}_{\text {acc }}$ plotted against the mean value of the distance for each subsample. It is evident that at smaller distances from the central star sources appear to have a higher mean $\dot{M}_{\text {acc }}$ that decreases monotonically with the distance. This result, however, does not necessarily indicate a real trend. In fact, around the Trapezium cluster, the detection limit is generally poorer than at larger distances, due to the 


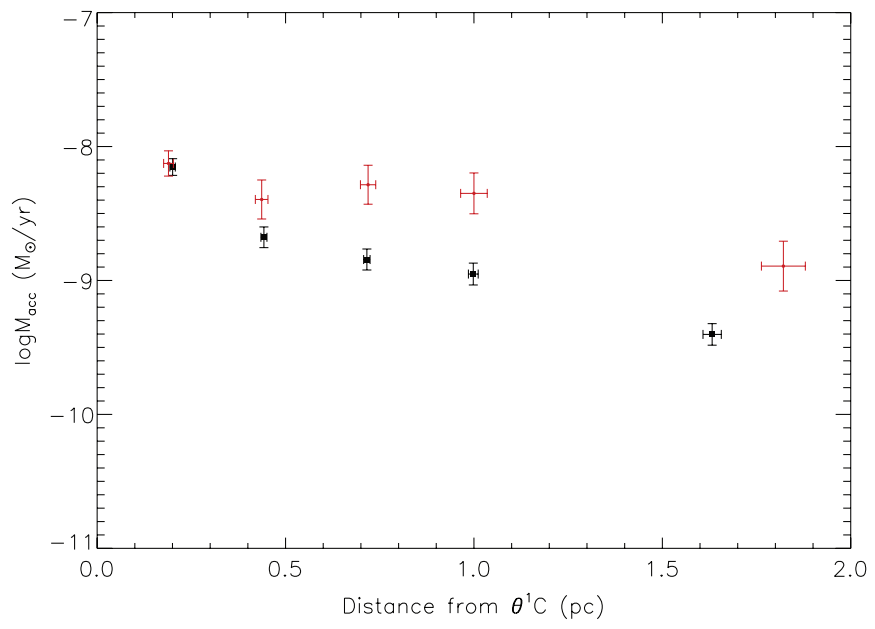

Figure 13. Mean values of $\dot{M}_{\mathrm{acc}}$ at increasing distances from $\theta^{1}$ Orionis C. The black points represent the values using all the sources in the sample and dividing them in five subsamples of equal number of sources. Red points represent the mean values when selecting for each subsample only the sources with $M_{*}>0.3 M_{\odot}$ and $5.5<\log (\mathrm{age} / \mathrm{yr})<7.3$. In the first case a trend of lower $\dot{M}_{\text {acc }}$ at higher distances is clear, but can be due, at least partially, to selection effects, as we show with the red points, where the distribution is flatter.

(A color version of this figure is available in the online journal.)

higher nebular background. Therefore, low-mass stars (whose $\dot{M}_{\text {acc }}$ are generally lower, as described in the previous sections) are less easily detected. Therefore, we select in each subsample only the sources with $M_{*}<0.3 M_{\odot}$ and with $5.5<\log (t / \mathrm{yr})<$ 7.3 , to have similar selection effect in each bin. The result is shown with the red circles in Figure 13: the values of $\dot{M}_{\text {acc }}$ as a function of distance have a much flatter distribution. Only at distances $\gtrsim 1.5 \mathrm{pc}$ the mean value of $\dot{M}_{\text {acc }}$ is lower.

\section{CONCLUSION}

We have presented a study of mass accretion rates $\left(\dot{M}_{\text {acc }}\right)$ in the ONC, for an unprecedentedly large ( $\sim 700$ stars $)$ sample of PMS stars. This allowed us to perform a thorough statistical analysis of the dependence of this quantity on the central stellar parameters, and investigate this phase of the stellar mass buildup and disk evolution.

We based our study on $H S T /$ WFPC2 photometric data over a large field of view and derived $\dot{M}_{\text {acc }}$ using two different accretion tracers: the $(U-B)$ excess and the $L_{\mathrm{H} \alpha}$. We study the systematic dependence of $\dot{M}_{\text {acc }}$ with respect to the age of the sources, their mass, and position within the region. In particular, the $\dot{M}_{\text {acc }}$ is found to vary with age and mass altogether. Our final relation between all these quantities is given by Equation (6) and in Table 6, and our best-fit relation using all the sources in the sample is given by $\log \left(\dot{M}_{\text {acc }} / M_{\odot}\right.$ yr $)=(-5.12 \pm 0.86)-$ $(0.46 \pm 0.13) \cdot \log (t / \mathrm{yr})-(5.75 \pm 1.47) \cdot \log \left(M_{*} / M_{\odot}\right)+$ $(1.17 \pm 0.23) \cdot \log (t / \mathrm{yr}) \cdot \log \left(M_{*} / M_{\odot}\right)$. We clearly find that the $\dot{M}_{\text {acc }}$ increases with stellar mass and decreases over evolutionary time.

Interestingly, we also find evidence that for more massive stars the decay of $\dot{M}_{\text {acc }}$ with time is much slower than for lower stellar masses. Similarly, for older stars, the dependence of $\dot{M}_{\text {acc }}$ with $M_{*}$ appears significantly steeper. This might imply that these objects are not in the asymptotic regime (i.e., when $t \gg t_{v}$, where $\dot{M}_{\text {acc }} \propto t^{-\eta}$ ), or that the hypothesis of a simple dependence of the viscosity $v \propto R^{\gamma}$ is probably not compatible with our observations. In particular, we suggest significant

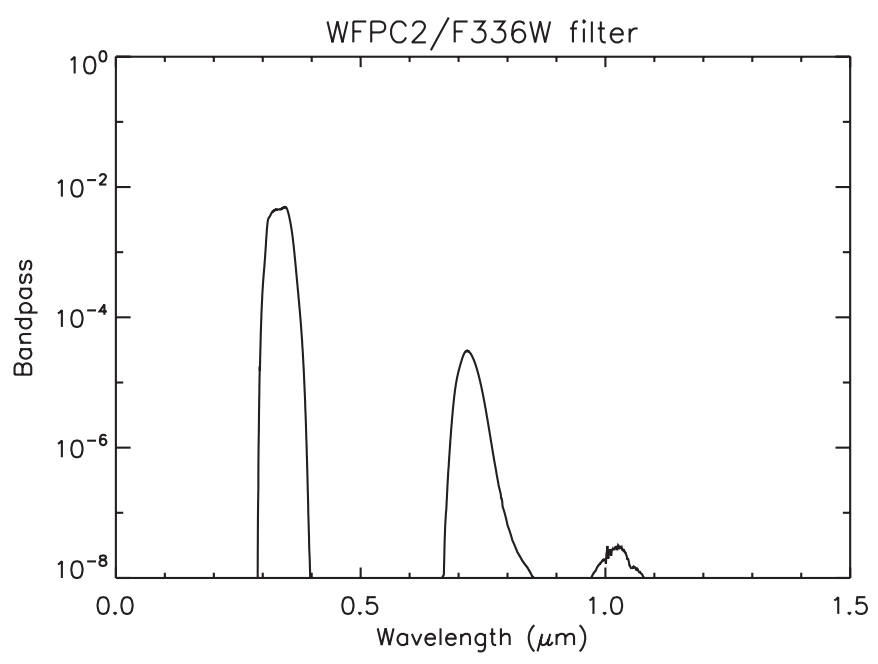

Figure 14. Bandpass of the filter $F 336 \mathrm{~W} / \mathrm{WFPC} 2$. Note the red leak component at $\sim 0.75 \mu \mathrm{m}$, where the filter has a window of transmission with $1 \%$ of the peak transmission at $\sim 0.35 \mu \mathrm{m}$.

discrepancies of our results with respect to the self-similar parameterization for sources with masses higher than $\sim 0.5 M_{\odot}$.

This work was made possible by GO program 10246 of the Hubble Space Telescope, which is operated by the Space Telescope Science Institute. C.F.M. thanks James Pringle and Philip J. Armitage for insightful discussions.

Facility: HST (WFPC2)

\section{APPENDIX A \\ RED LEAK}

As mentioned in Section 2.1, the filter $F 336 W$ of WFPC2 is affected by red leak. This is evident from Figure 14, where the band profile is shown. At $\lambda \sim 7300 \AA$, the filter shows a transmission window whose peak throughput is $\sim 0.5 \%$ that in the $U$-band wavelength range. There is also a third peak at $\sim 1 \mu \mathrm{m}$, but this is completely negligible, since its bandpass is less than $10^{-5}$ of the main peak and the detector quantum efficiency is nearly zero.

The presence of red leak in $F 336 W$ does not significantly affect the stellar photometry of blue sources, as most of the flux is emitted at short wavelengths. For red objects, however, the leak contribution may be significant and, in extreme cases, even dominant. In order to accurately account for the $U$-band fluxes of our ONC sources, and prevent introducing a systematic overestimate of the accretion luminosity $\left(L_{\text {acc }}\right)$ and mass accretion rate $\left(\dot{M}_{\mathrm{acc}}\right)$, we must estimate and correct for the spurious red-leak excess.

The number of photons collected in the leaking part of the filter depends solely on the observed flux at the wavelength of the leak (referred to as $U_{\text {leak }}$, in magnitudes). To estimate this flux, we considered our WFPC2 I-band photometry and computed the color term $\left(U_{\text {leak }}-I\right)$ by means of synthetic photometry. As this color term depends mainly on $T_{\text {eff }}$ and $A_{V}$, we computed it for each source considering the stellar parameters from Da Rio et al. (2012), and assuming the atmosphere models from Allard et al. (2011), corrected as explained in Appendix B.

We then converted $U_{\text {leak }}$ from magnitudes to photons $\mathrm{s}^{-1}$. To this end, one must derive the zeropoint of the leak window of the filter, i.e., the number of photons passing through the leak for a magnitude $m=0$. This zero point is simply the 
zero point of the whole $F 336 W$ filter ( $\mathrm{ZP}_{\mathrm{F} 336 \mathrm{~W}}$; Dolphin 2009), scaled to a term equal to the fraction of $U$-band photons that pass through the leak. We derived this latter term from the calibrated spectrum of Vega of Bohlin (2007) and the measured $F 336 W$ throughput. We find that the fraction of photons in the red leak part of the spectrum of Vega is $0.551 \%$ of the total number of $U$-band photons. Therefore, we obtain counts $_{\text {leak }}=10^{-0.4\left(U_{\text {leak }}-\mathrm{ZP}\right)} \cdot 0.00551$.

Finally, we considered our observed $U$ magnitudes, converted them in units of counts (using again $\mathrm{ZP}_{F 336 W}$ ), subtracted the leak contribution, and transformed them once again in magnitudes. The result is the leak-corrected $U$-band magnitude, or $U_{\text {noleak }}$. The leak offset in magnitude, for our stellar sample, has a mean value of about $0.5 \mathrm{mag}$. We refer in all the text to the $U$ band as the corrected one, using the extended notation $U_{\text {noleak }}$ only when needed, for clarity.

\section{APPENDIX B}

\section{ISOCHRONE CALIBRATION}

In order to calibrate the appropriate isochrone for our sample, we start by considering a family of synthetic spectra, the BT-SETTL from Allard et al. (2011). As shown in their work, these models seem to reproduce the observed colors of stars and BDs in the $J$ and $K$ bands. However, their validity in the optical range has not been demonstrated. We therefore test their accuracy taking advantage of the $J$-band photometry on the ONC from Robberto et al. (2010). The concept is the following: assuming the validity of the predicted $J$-band colors as a function of $T_{\text {eff }}$, if the predicted $I$-band colors are also correct, then the synthetic $(I-J)$ should also agree with the data. Otherwise, a correction to the synthetic I-band magnitude is required. Subsequently, the same procedure can iterated to the $B$ and $U$ bands as well.

Figure 15 illustrates this test: the upper panel shows the observed (gray open circles) and extinction-corrected (black dots) photometry of our ONC members, assuming the $A_{V}$ from Da Rio et al. (2012), and limited to the candidate weak accretors (Da Rio et al. 2010, 2009), i.e., sources with $\log \left(L_{\text {acc }} / L_{\text {tot }}\right)_{\text {DaRio10 }}<$ -1.5 and $\mathrm{EW}_{\mathrm{H} \alpha, \text { DaRio09 }}<10 \AA$. Under these assumptions, the black dots, therefore, trace the photospheric colors of ONC sources of different temperatures. The red dashed line represents a $1 \mathrm{Myr}$ synthetic isochrone from the models of Baraffe et al. (1998), converted into colors assuming the BT-SETTL atmosphere models. We find that the predicted colors are systematically underestimated for $T_{\text {eff }} \lesssim 3200 \mathrm{~K}$. We thus interpolate the empirical $I-J$ color locus (blue solid line) and determine the offset between the two. We calibrated the $I$-band magnitudes of the isochrone as a function of $T_{\text {eff }}$, by applying this correction to the synthetic $I$-band magnitudes.

From the calibrated $I$-band photospheric magnitudes, we iterate the same procedure first to the $B-I$ colors, in order to calibrate $B$ (Figure 15, middle panel), then to the $U-B$ color, to calibrate the intrinsic $U$ magnitude (lower panel). The selection of the candidate non-accretors is particulary critical for these two bands, which are highly influenced by accretion excesses.

We stress that for practical reasons we performed a calibration of the synthetic isochrone in terms of magnitudes, but for our purposes (the analysis of our 2CD) only the accuracy of the colors as a function of temperature are relevant. Also, colors are independent on distance or small differences in stellar radii, therefore a luminosity spread for ONC members, due to, e.g., a spread in stellar ages, does not affect our method. We recap the

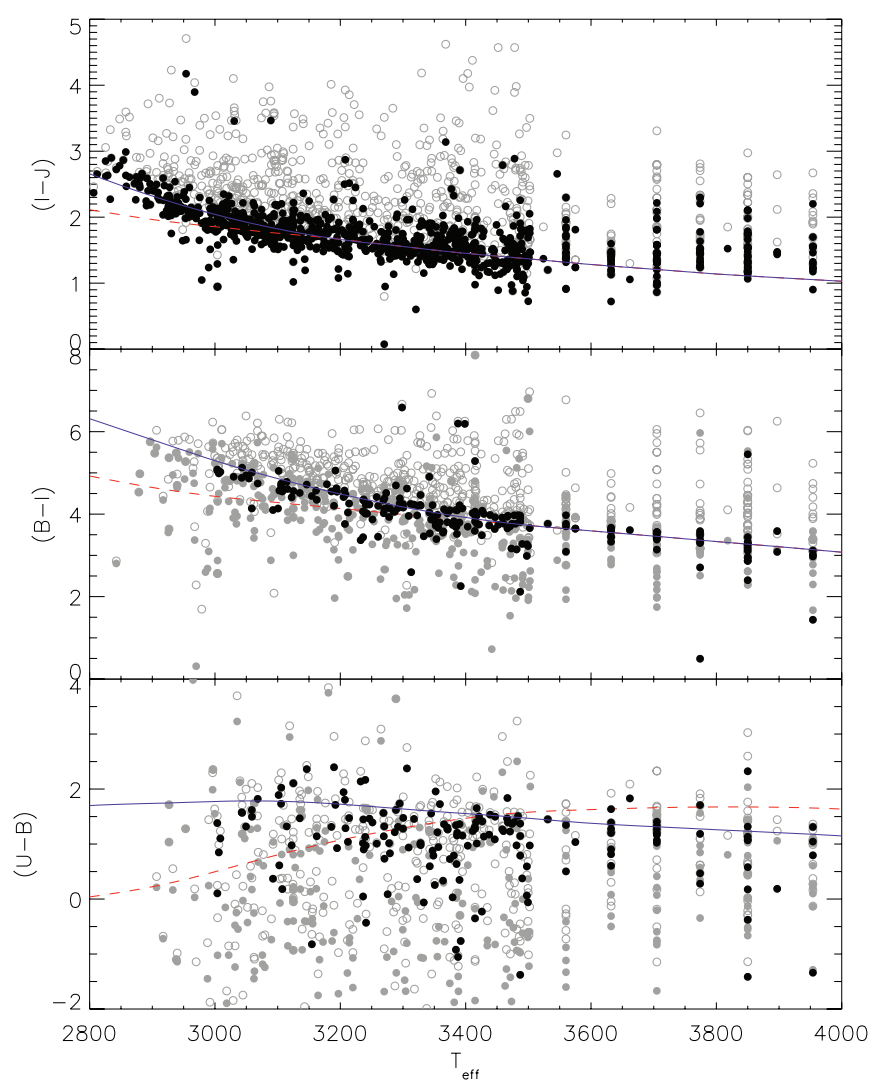

Figure 15. Procedure used to calibrate the models by Allard et al. (2011) in our photometric bands. The red dashed line represents the color computed through synthetic photometry on the BT-SETTL models. Gray open circles are the observed colors of the sources, gray filled ones the corrected values according to $A_{V}$ from Da Rio et al. (2012) and black filled points are corrected values of low accretors according to Da Rio et al. (2010). We use the latter ones to determine the empirical calibration, which is the blue line.

(A color version of this figure is available in the online journal.)

empirical estimate of the WFPC2 colors found for the calibration of the BT-SETTL models in Table 1.

\section{REFERENCES}

Alexander, R. D., \& Armitage, P. J. 2006, ApJ, 639, L83

Allard, F., Homeier, D., \& Freytag, B. 2011, in ASP Conf. Ser. 448, 16th Cambridge Workshop on Cool Stars, Stellar Systems, and the Sun, ed. C. Johns-Krull (San Francisco: ASP), 91

Balbus, S. A., \& Hawley, J. F. 1992, ApJ, 400, 610

Baraffe, I., \& Chabrier, G. 2010, A\&A, 521, A44

Baraffe, I., Chabrier, G., Allard, F., \& Hauschildt, P. H. 1998, A\&A, 337, 403

Baraffe, I., Chabrier, G., \& Gallardo, J. 2009, ApJ, 702, L27

Beccari, G., Spezzi, L., De Marchi, G., et al. 2010, ApJ, 720, 1108

Binney, J., \& Tremaine, S. 2008, in Galactic Dynamics, ed. J. Binney \& S. Tremaine (2nd ed.; Princeton: Princeton Univ. Press), 771

Bohlin, R. C. 2007, in ASP Conf. Ser. 364, The Future of Photometric, Spectrophotometric and Polarimetric Standardization, ed. C. Sterken (San Francisco, CA: ASP), 315

Calvet, N., \& Gullbring, E. 1998, ApJ, 509, 802

Cardelli, J. A., Clayton, G. C., \& Mathis, J. S. 1989, ApJ, 345, 245

Clarke, C. J., \& Pringle, J. E. 2006, MNRAS, 370, L10

Costero, R., \& Peimbert, M. 1970, Bol. Obs. Tonantzintla Tacubaya, 5, 229

Da Rio, N., Robberto, M., Hillenbrand, L. A., Henning, T., \& Stassun, K. G. 2012, ApJ, 748, 14

Da Rio, N., Robberto, M., Soderblom, D. R., et al. 2009, ApJS, 183, 261

Da Rio, N., Robberto, M., Soderblom, D. R., et al. 2010, ApJ, 722, 1092

Dahm, S. E. 2005, PhD thesis, Univ. Hawaii

Dahm, S. E. 2008, AJ, 136, 521

D’Antona, F., \& Mazzitelli, I. 1994, ApJS, 90, 467

De Marchi, G., Panagia, N., \& Romaniello, M. 2010, ApJ, 715, 1 
De Marchi, G., Panagia, N., Romaniello, M., et al. 2011, ApJ, 740, 11 Dolphin, A. E. 2009, PASP, 121, 655

Fedele, D., van den Ancker, M. E., Henning, T., Jayawardhana, R., \& Oliveira, J. M. 2010, A\&A, 510, A72

Ferland, G. J., Korista, K. T., Verner, D. A., et al. 1998, PASP, 110, 761

Fischer, W., Edwards, S., Hillenbrand, L., \& Kwan, J. 2011, ApJ, 730, 73

France, K., Yang, H., \& Linsky, J. L. 2011, ApJ, 729, 7

Gammie, C. F. 1996, ApJ, 457, 355

Gullbring, E., Calvet, N., Muzerolle, J., \& Hartmann, L. 2000, ApJ, 544, 927

Gullbring, E., Hartmann, L., Briceno, C., \& Calvet, N. 1998, ApJ, 492, 323

Haisch, K. E., Jr., Lada, E. A., \& Lada, C. J. 2001, ApJ, 553, L153

Hartmann, L., Calvet, N., Gullbring, E., \& D’Alessio, P. 1998, ApJ, 495, 385

Hartmann, L., Cassen, P., \& Kenyon, S. J. 1997, ApJ, 475, 770

Herczeg, G. J., \& Hillenbrand, L. A. 2008, ApJ, 681, 594

Hillenbrand, L. A. 1997, AJ, 113, 1733

Hosokawa, T., Offner, S. S. R., \& Krumholz, M. R. 2011, ApJ, 738, 140

Lodato, G. 2008, New Astron. Rev., 52, 21

Lodato, G., \& Rice, W. K. M. 2004, MNRAS, 351, 630

Lynden-Bell, D., \& Pringle, J. E. 1974, MNRAS, 168, 603

Menten, K. M., Reid, M. J., Forbrich, J., \& Brunthaler, A. 2007, A\&A, 474, 515

Mortier, A., Oliveira, I., \& van Dishoeck, E. F. 2011, MNRAS, 418, 1194

Murphy, S. J., Lawson, W. A., Bessell, M. S., \& Bayliss, D. D. R. 2011, MNRAS, 411, L51
Muzerolle, J., Calvet, N., \& Hartmann, L. 2001, ApJ, 550, 944

Muzerolle, J., Hillenbrand, L., Calvet, N., Briceño, C., \& Hartmann, L. 2003, ApJ, 592, 266

Natta, A., Testi, L., \& Randich, S. 2006, A\&A, 452, 245

Palla, F., \& Stahler, S. W. 1999, ApJ, 525, 772

Reggiani, M., Robberto, M., da Rio, N., et al. 2011, A\&A, 534, A83

Ricci, L., Robberto, M., \& Soderblom, D. R. 2008, AJ, 136, 2136

Rigliaco, E., Natta, A., Randich, S., Testi, L., \& Biazzo, K. 2011, A\&A, 525, A47

Robberto, M., Soderblom, D. R., Scandariato, G., et al. 2010, AJ, 139, 950

Robberto, M., Song, J., Mora Carrillo, G., et al. 2004, ApJ, 606, 952

Robberto, M., et al. 2012, ApJS, submitted

Shu, F., Najita, J., Ostriker, E., et al. 1994, ApJ, 429, 781

Sicilia-Aguilar, A., Henning, T., \& Hartmann, L. W. 2010, ApJ, 710, 597

Siess, L., Dufour, E., \& Forestini, M. 2000, A\&A, 358, 593

Skemer, A. J., Close, L. M., Szű́cs, L., et al. 2011, ApJ, 732, 107

Spezzi, L., de Marchi, G., Panagia, N., Sicilia-Aguilar, A., \& Ercolano, B. 2012, MNRAS, 421, 78

Tilling, I., Clarke, C. J., Pringle, J. E., \& Tout, C. A. 2008, MNRAS, 385, 1530

Valenti, J. A., Basri, G., \& Johns, C. M. 1993, AJ, 106, 2024

White, R. J., \& Basri, G. 2003, ApJ, 582, 1109 